\title{
DYNAMICS OF THREE COUPLED VAN DER POL OSCILLATORS WITH APPLICATION TO CIRCADIAN RHYTHMS
}

\author{
A Thesis \\ Presented to the Faculty of the Graduate School \\ of Cornell University \\ in Partial Fulfillment of the Requirements for the Degree of \\ Master of Science
}

by

Kevin Robert Rompala

May 2006 
(c) 2006 Kevin Robert Rompala

\section{ALL RIGHTS RESERVED}




\begin{abstract}
In this thesis we study the dynamics of the in-phase mode for a system of three coupled van der Pol oscillators. Two of the oscillators are taken to be identical and coupled indirectly via a third oscillator whose natural frequency may vary. We use the singular perturbation method known as two-variable expansion to obtain a slow-flow, which is then analyzed using the computer algebra system MACSYMA. We find analytical representations for saddle-node and Hopf bifurcation curves in the parameter space and explore the dynamics found in the in-phase phase space.

The motivation for this work comes from the presence of circadian melatonin rhythms in the eyes of Japanese quail. Recent experiments showing the rhythms in the eyes to be tightly coupled and in-phase with each other have strengthened the hypothesis that the eyes are the location of the central pacemaker for Japanese quail. The melatonin rhythm in each eye is modeled as a van der Pol limit-cycle oscillator. Furthermore, the eyes cannot directly communicate to each other, but do so via a connection to an extra-ocular circadian system, here represented by the third oscillator.
\end{abstract}




\section{BIOGRAPHICAL SKETCH}

Kevin Robert Rompala was born on December 12, 1980 in Chicago, Illinois. He graduated second in his class at Lake Park High School in Roselle, IL in 1999. In August 1999 he began attending Washington University in St. Louis, where he graduated from in May 2003 with a Bachelor of Science degree in Systems Science

\& Engineering. That fall he entered the Department of Theoretical \& Applied Mechanics at Cornell University where he is currently pursuing his doctorate. 
This thesis is dedicated to my parents, Robert and Linda Rompala, and to Katie Stull, with whom I happily share my life. 


\section{ACKNOWLEDGEMENTS}

I would like to thank my thesis advisor and committee chairman, Richard Rand, for bringing this project to my attention and for his encouragement and support throughout the first half of my graduate studies. His patience with a graduate student whose research progresses on multiple timescales is greatly appreciated. I would also like to thank Howard Howland for serving on my committee. 


\section{TABLE OF CONTENTS}

1 Introduction 1

2 Derivation of the Slow-Flow Equations 8

2.1 Slow-Flow in Rectangular Coordinates . . . . . . . . . . . . . . . . 11

2.2 Slow-Flow via Method of Averaging . . . . . . . . . . . . . . . . . . 12

3 Investigation of the Parameter Space for the In-Phase Mode 17

3.1 Saddle-Node Bifurcation Curves . . . . . . . . . . . . . . . . . 18

3.2 Hopf Bifurcation Curves . . . . . . . . . . . . . . . . . . 22

3.3 Other Bifurcation Curves . . . . . . . . . . . . . . . . 27

4 The Dynamics of the In-Phase Mode 29

4.1 Transitions Between Regions of Parameter Space . . . . . . . . . . 31

4.2 Analysis of the Slow-Flow Dynamics . . . . . . . . . . . . . . . . 43

5 The Slow-Flow on Singular Surfaces $\quad \mathbf{4 5}$

5.1 Slow-Flow on the Singular Surface $R_{1}=0$. . . . . . . . . . . 45

5.2 Slow-Flow on the Singular Surface $R_{3}=0$. . . . . . . . . . . . . . 48

5.3 Further Analysis of the Singular Dynamics . . . . . . . . . . . 51

6 Conclusions $\quad 55$

$\begin{array}{ll}\text { Bibliography } & 62\end{array}$ 


\section{LIST OF FIGURES}

1.1 Possible model for the multioscillatory circadian system of the Japanese quail (extra-ocular photoreceptors not shown). RHT = retinohypothalamic tract. . . . . . . . . . . . . . . 3

1.2 Proposed model for the interaction of the occular and pineal oscillators. . . . . . . . . . . . . . . . . . 6

1.3 Previously proposed model for the interaction of the occular and pineal oscillators. . . . . . . . . . . . . . . . . . 7

3.1 Saddle-node bifurcation curves in parameter space. . . . . . . . . . 21

3.2 Hopf (plus saddle-node) bifurcation curves in parameter space. . . 26

3.3 Numerically computed bifurcation curves (dotted lines) in parameter space. . . . . . . . . . . . . . . . . . . . 27

3.4 Final numerical bifurcation curves (dashed lines) in parameter space. 28

4.1 Three distinct types of dynamical behavior for the slow-flow. . . . 30

4.2 Regions of parameter space exhibiting distinctly different dynamical behavior. . . . . . . . . . . . . . . . . . . 32

4.3 Path followed when crossing from Region I into Region III and then into Region IV. . . . . . . . . . . . . . . . . . . . . . . . . . . . . 33

4.4 2-dimensional cartoon of 3-dimensional bifurcations: (a) Two unstable equilibrium points separated by a stable LCD. (b) An infiniteperiod bifurcation occurs along stable LCD creating new stable and unstable equilibrium points. Dashed line represents structurally unstable (bifurcating) LCD. (c) Two unstable equilibrium points converge on each other. (d) A saddle-node bifurcation occurs between two unstable equilibrium points. (e) One stable and one unstable equilibrium point remain. . . . . . . . . . . . . . . . . 34

4.5 Path followed when crossing from Region I into Region D and then into Region IV. . . . . . . . . . . . . . . . . . . . . . . . . . . . 36

4.6 2-dimensional cartoon of 3-dimensional bifurcations: (a) Two unstable equilibrium points separated by a stable LCD. (b) A heteroclinic (saddle-connection) bifurcation occurs. Dashed line represents structurally unstable (bifurcating) LCD. (c) After the saddleconnection bifurcation the stable LCD becomes a stable LCW surrounding an unstable equilibrium. (d) The stable LCW decreases in size until a super-critical Hopf bifurcation happens. (e) One stable and one unstable equilibrium point remain. . . . . . . . . . . 37

4.7 Path followed when crossing from Region IV into Region $\mathrm{V}$ and then into Region VI. . . . . . . . . . . . . . . . . . . . . . 38 
4.8 2-dimensional cartoon of 3-dimensional bifurcations: (a) Two stable equilibrium points separated by an unstable LCD. (b) An infiniteperiod bifurcation occurs along the unstable LCD creating two new unstable equilibrium points. Dashed line represents structurally unstable (bifurcating) LCD. (c) A stable and unstable equilibrium point converge on each other. (d) A saddle-node bifurcation occurs between a stable and unstable equilibrium point. (e) One stable and one unstable equilibrium point remain. . . . . . . . . . . . .

4.9 Path followed when crossing from Region IV into Region $\mathrm{C}$ and then into Region VI. . . . . . . . . . . . . . . . . . . . . . . . . .

4.10 2-dimensional cartoon of 3-dimensional bifurcations: (a) One stable and one unstable equilibrium point exist. (b) A fold of LCWs occurs around the unstable equilibrium point. Dashed line represents structurally unstable (bifurcating) LCW. (c) As the LCWs separate, the stable LCW shrinks in size while the unstable LCW grows. (d) A heteroclinic (saddle-connection) bifurcation occurs. Dashed line represents structurally unstable (bifurcating) LCW. (e) A Hopf bifurcation occurs as the stable LCW collapses to the unstable equilibrium point. (f) Two stable equilibria exist and are separated by an unstable LCD. . . . . . . . . . . . . . . . . . . . 42

4.11 Regions of parameter space where stable phase-locked motion is possible. . . . . . . . . . . . . . . . 44

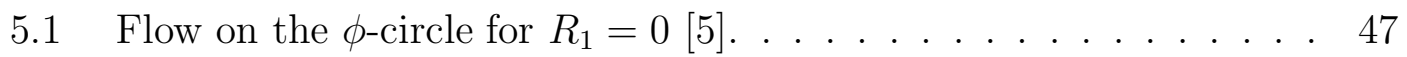

5.2 Flow on the $\phi$-circle for $R_{3}=0$. . . . . . . . . . . . . . . 50

5.3 Examples of flows on the two singular planes $R_{1}=0$ and $R_{3}=0 . \quad$. 52

5.4 Example of stable negative drift when $(\Delta, \mu)=(0.2,0.3024) \ldots$. . 53

5.5 Example of stable LCW when $(\Delta, \mu)=(0.2,0.304) \ldots . . . . \quad 54$

6.1 Bifurcation curves in parameter space. . . . . . . . . . . . 56

6.2 Regions of parameter space where stable phase-locked motion is possible. . . . . . . . . . . . . . . 56

6.3 Bifurcation curves for two weakly-coupled van der Pol oscillators (modified from [4], [5]) . . . . . . . . . . . . . . 58

6.4 Regions of bi-stability (shown in black) for two weakly-coupled van der Pol oscillators . . . . . . . . . . . . . . . . . 59

6.5 Regions of bi-stability (shown in black) for the slow-flow (3.3). . . 60

6.6 Saddle-node bifurcation curves in parameter space associated with equation (6.2) for different values of $N$ : (A) $N=2$, (B) $N=1.5$, (C) $N=1.25,($ D) $N=1.1 . \ldots \ldots \ldots$ 


\section{CHAPTER 1 INTRODUCTION}

In this thesis we model a portion of the multioscillatory circadian system of the Japanese quail. We hypothesize that the endogenous melatonin rhythms present in the eyes act as circadian pacemakers and are indirectly coupled to each other through an extra-ocular, melatonin producing circadian system. This extra-ocular system comprises oscillators found in the pineal gland (that rhythmicly produce and secrete melatonin into the bloodstream) and hypothalamic areas of the brain. The model builds on an earlier attempt to model the coupling between the eyes via a melatonin "bath" [1], [2].

The circadian system of all avian species consists of three primary components (see Figure 1.1): the pineal gland which rhythmicly produces and secretes melatonin, a hypothalamic oscillator (presumably located in the suprachiasmatic nucleus, or SCN) that most likely acts through neural signals, and the retinae of the eyes, which act through hormonal (melatonin) and/or neural outputs (see [13] for a review). Experimental evidence shows the relative importance of these components in generating circadian rhythms varies from species to species. In some passerine birds, such as the house sparrow or European starling, the pineal appears to be the only source of plasma melatonin and the pacemaker for the entire circadian system as pinealectomy abolishes any plasma melatonin rhythms and many behavioral patterns [7]. In other non-passerine birds such as the pigeon and Japanese quail the eyes are also sources of plasma melatonin [12]. In the pigeon, both pinealectomy and eye removal are necessary to abolish overt behavior rhythms. In the quail, eye removal alone causes arrhythmia in body temperature [10]. 
Two different models of the organization of the avian circadian system have been proposed in recent literature. First, the pineal gland and the SCN have been conjectured to be joined together in a "neuroendocrine loop" [3] in which "a daily melatonin output from the pineal is required to maintain rhythmicity in the SCN, and a daily neural output from the $\mathrm{SCN}$ is required to maintain rhythmicity in the pineal." [10] This relationship between the pineal and SCN is mutually inhibitory - during night the pineal produces and secretes melatonin into the bloodstream which inhibits SCN activity and during the day the SCN inhibits the pineal through neural signals. From this model we can expect the pineal and SCN rhythms to be 180 degrees out-of-phase. A second model dubbed the "internal resonance model" hypothesizes that the pineal and SCN maintain a strong circadian rhythm through resonance [6]. In both these models it is assumed that the pineal and SCN oscillators are damped oscillators that maintain their mutual rhythms through coupling.

Complete removal of the eyes causes loss of rhythmicity of body temperature in Japanese quail, a rhythm thought to be controlled by the SCN [10]. Thus the eyes appear to be essential to the sustainment of circadian rhythms in the SCN in this species. Most likely this is accomplished via both neural (the nature of which is still unknown) and hormonal outputs. Evidence suggesting a hormonal component includes the fact that severing the optic nerve only produces arrhythmia in approximately $25 \%$ of Japanese quail undergoing the procedure [10].

Recent experiments [10] have strengthened the conclusion that the circadian rhythms present in the eyes are the central pacemakers for the entire circadian system in 


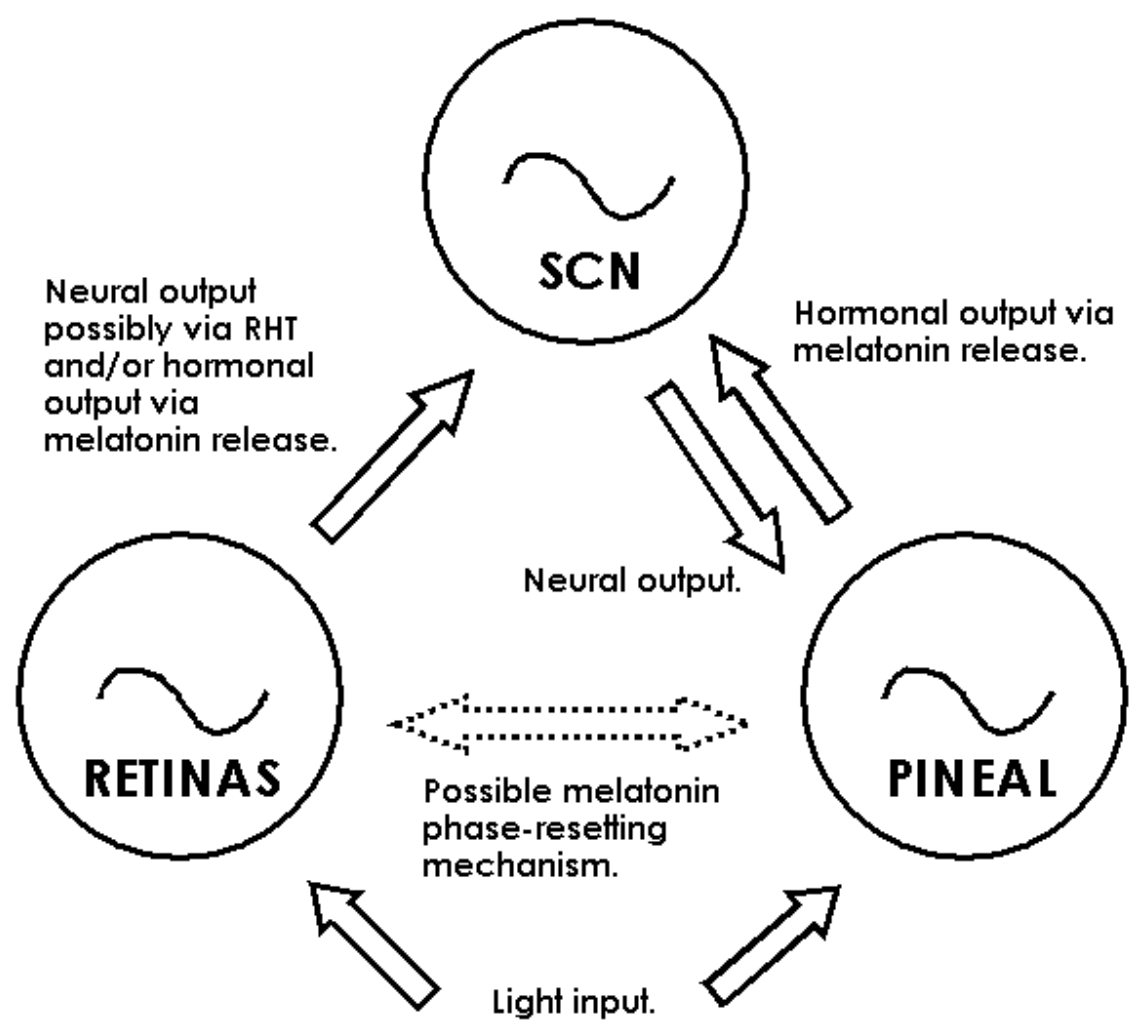

Figure 1.1: Possible model for the multioscillatory circadian system of the Japanese quail (extra-ocular photoreceptors not shown). RHT = retinohypothalamic tract.

Japanese quail. The authors state

...if the eyes are indeed the major circadian pacemakers in this system, the two ocular pacemakers in an individual bird must remain in phase with each other in prolonged DD [constant darkness]. If the two ocular pacemakers do not maintain phase, it would be difficult to argue that they are responsible for driving the robust, persistent body temperature rhythm that is characteristic of quail free-running in DD. 
Their experiments showed that when Japanese quail are placed in constant darkness (representing an autonomous system with no external forcing), the level of melatonin in their two eyes are found to be in phase after a period of more than 40 days. Furthermore, experiments showed that this in-phase stability is robust and seems to hold for a wide range of initial conditions, even, e.g., if the two eyes are initially light-loaded to be out of phase.

In this thesis we model a small subset of the entire avian circadian system to see if we can duplicate the experimentally observed stability of the in-phase melatonin oscillations in the eyes under constant conditions. We focus on only phase-locked, or entrained, retinal and extra-ocular melatonin rhythms. In Japanese quail, both the retinae and the pineal gland rhythmically secrete melatonin into the bloodstream and therefore both can be involved in sustaining the rhythm of the SCN. Thus in order for these separate rhythms to produce a singular pacemaker rhythm we require them to at least be phase-locked. Since this model consists of only a subset of the circadian system we make the assumptions that the SCN of the chick and the pineal gland remain intact (and are included in the extra-ocular circadian system) and that we are considering only free-running behavior (represented by the autonomous nature of the equations in (1.1)). Furthermore we ignore any possible neural coupling between the eyes via the SCN or any other neurological connections, as this would necessitate including the SCN separately in our model.

Our hypothesized model for the quail circadian system consists of three coupled nonlinear van der Pol oscillators. The van der Pol oscillators were chosen because (i) each oscillator exhibits a self-sustaining oscillation (see [9] for review) and (ii) 
it has been shown [2] that a simple model for the biochemical basis of a circadian rhythm in free-running conditions can be reduced to such an oscillator.

The eyes are represented by identical (same natural frequency) oscillators, $x$ and $y$, and the extra-ocular circadian system by a third oscillator, $w$, whose natural frequency is allowed to vary slightly from that of the other oscillators. The eye oscillators are indirectly coupled via the extra-ocular system (see Figure 1.2). While the true nature of the coupling between the eyes is still unknown, the retinae do possess melatonin receptors so that one could hypothesize that "if the eyes are driven out of phase, daily melatonin release by the eyes (or pineal) may cause mutual phase advances or delays of the two ocular pacemakers until they regain their normal phase relationship." [10] The system can be represented as

$$
\begin{gathered}
\ddot{x}-\epsilon\left(1-x^{2}\right) \dot{x}+x=\epsilon \mu(w-x) \\
\ddot{y}-\epsilon\left(1-y^{2}\right) \dot{y}+y=\epsilon \mu(w-y) \\
\ddot{w}-\epsilon\left(1-w^{2}\right) \dot{w}+p^{2} w=\epsilon \mu(x+y-2 w)
\end{gathered}
$$

where $p=1+\epsilon \Delta, \mu$ represents the coupling strength between the oscillators, and $\epsilon \ll 1$

A previous attempt at modeling this behavior considered the melatonin rhythms in the eyes to be coupled via a "bath" [2], [1]. The premise was that eyes "communicate" by altering the concentration of melatonin present in the bloodstream, a.k.a. the "bath" (see Figure 1.3). Here, the eyes, with the non-oscillatory bloodstream, were modeled as

$$
\frac{d^{2} x}{d t^{2}}-\epsilon\left(1-x^{2}\right) \frac{d x}{d t}+x=k(z-x)
$$




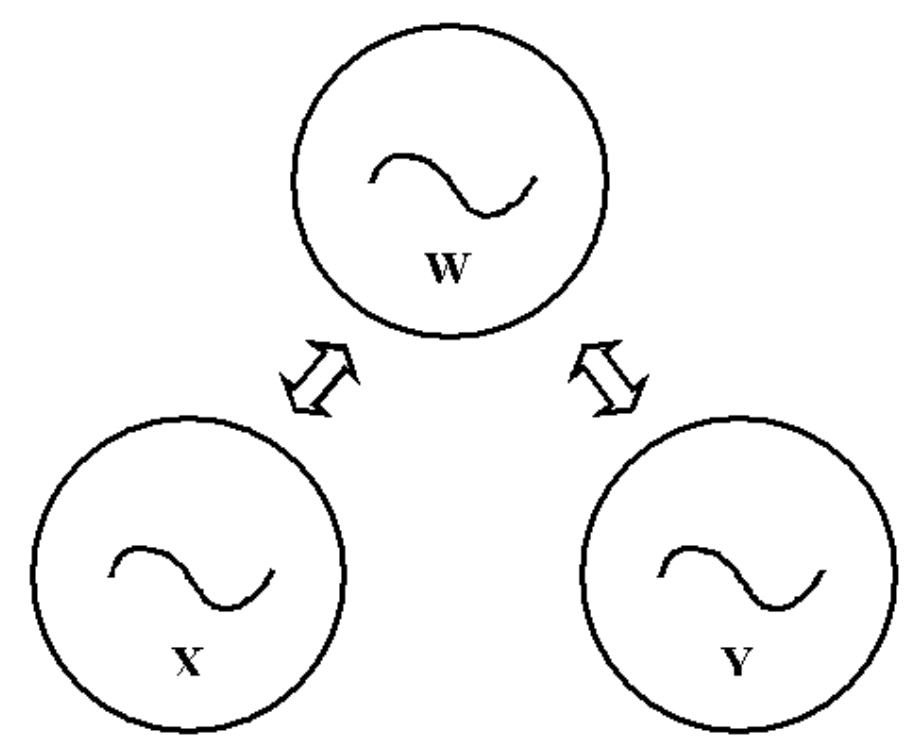

Figure 1.2: Proposed model for the interaction of the occular and pineal oscillators.

$$
\begin{gathered}
\frac{d^{2} y}{d t^{2}}-\epsilon\left(1-y^{2}\right) \frac{d y}{d t}+y=k(z-y) \\
\frac{d z}{d t}=k(x-z)+k(y-z)
\end{gathered}
$$

with $k$ as the coupling strength and $\epsilon \ll 1$. Unfortunately, this model predicted the out-of-phase model to have greater stability than the in-phase mode, contradicting the experimental results.

The ultimate question we seek to answer concerning the model (1.1) is what are the stabilities of the in-phase and out-of-phase phase-locked modes of the oscillators found in the eyes? For what values of the parameters do they exist? For what values are they attracting (do motions eventually settle down to one of the modes after all transient behavior damps out)? Towards this end, we perform an analytical and numerical investigation of the in-phase mode of (1.1). The in-phase 


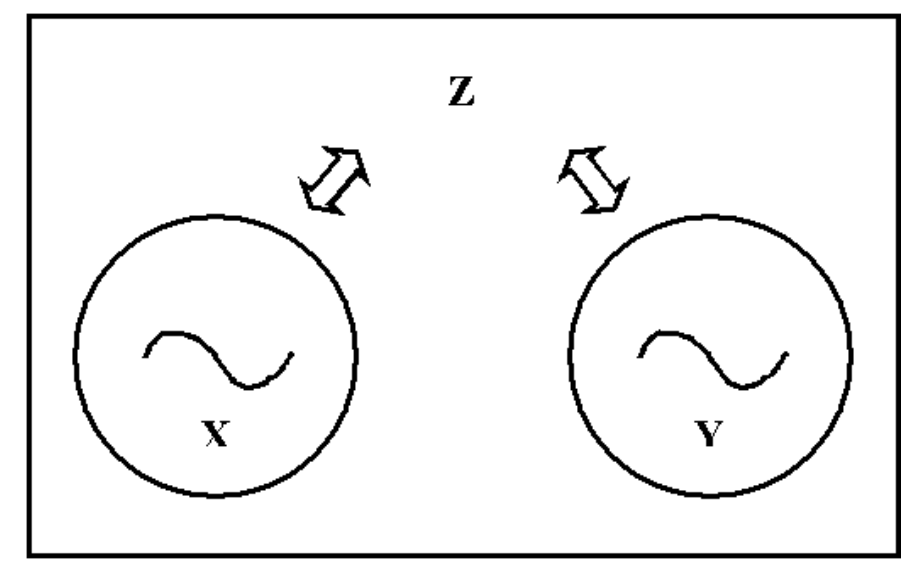

Figure 1.3: Previously proposed model for the interaction of the occular and pineal oscillators.

mode, $x=y$, satisfies the reduced system of equations

$$
\begin{gathered}
\frac{d^{2} x}{d t^{2}}-\epsilon\left(1-x^{2}\right) \frac{d x}{d t}+x=\epsilon \mu(w-x) \\
\frac{d^{2} w}{d t^{2}}-\epsilon\left(1-w^{2}\right) \frac{d w}{d t}+p^{2} w=\epsilon 2 \mu(x-w)
\end{gathered}
$$

Equations (1.3) represent a four-dimensional invariant manifold sitting inside the six-dimensional phase-space of (1.1). We ask for what values of the parameters $\Delta$ and $\mu$ does a stable periodic motion of (1.3) exist. We note that the ultimate question of stability in the larger six-dimensional space is not addressed in this thesis. 
CHAPTER 2

\section{DERIVATION OF THE SLOW-FLOW EQUATIONS}

The system of three weakly-coupled van der Pol oscillators under study is given as

$$
\begin{gathered}
\ddot{x}-\epsilon\left(1-x^{2}\right) \dot{x}+x=\epsilon \mu(w-x) \\
\ddot{y}-\epsilon\left(1-y^{2}\right) \dot{y}+y=\epsilon \mu(w-y) \\
\ddot{w}-\epsilon\left(1-w^{2}\right) \dot{w}+p^{2} w=\epsilon \mu(x+y-2 w)
\end{gathered}
$$

where the frequency of the $w$ oscillator is $p=1+\epsilon \Delta, \epsilon \ll 1$, and a "dotted" quantity implies differentiation with respect to time, $t$. The system depends on two parameters $-\mu$, the coupling strength between the oscillators, and $\Delta$, the detuning of the $w$ oscillator. Solution trajectories to (2.1) reside in a six-dimensional phase space. To analyze the dynamics of these weakly-coupled motions we use the singular perturbation method commonly referred to as two-variable expansion [9] to derive the slow-flow equations to $O(\epsilon)$. Algebraic computations and manipulations are performed using the computer algebra system MACSYMA.

To begin we define two new time scales for our solutions: slow-time, $\eta(t)$ and strained-time, $\zeta(t)$,

$$
\begin{aligned}
& \eta(t)=\epsilon t \\
& \zeta(t)=\omega t
\end{aligned}
$$

where $\omega=1+k_{1} \epsilon+O\left(\epsilon^{2}\right)$. The constant term $k_{1}$ builds the frequency-amplitude relationship that characterizes nonlinear dynamics into the solution and will (potentially) be determined during the elimination of secular terms. By considering the solutions to $(2.1)$ as functions of the two new time variables, i.e. $x(\eta, \zeta)$, the 
differential equations (2.1) must also be written in terms of these new variables. Taking the $x$ oscillator as an example, we differentiate using the chain-rule to get

$$
\begin{gathered}
\dot{x}=\frac{\partial x}{\partial \zeta} \dot{\zeta}+\frac{\partial x}{\partial \eta} \dot{\eta}=\omega \frac{\partial x}{\partial \zeta}+\epsilon \frac{\partial x}{\partial \eta} \\
\ddot{x}=\omega^{2} \frac{\partial^{2} x}{\partial \zeta^{2}}+2 \epsilon \omega \frac{\partial^{2} x}{\partial \zeta \partial \eta}+\epsilon^{2} \frac{\partial^{2} x}{\partial \eta^{2}}=\omega^{2} \frac{\partial^{2} x}{\partial \zeta^{2}}+2 \epsilon \omega \frac{\partial^{2} x}{\partial \zeta \partial \eta}+O\left(\epsilon^{2}\right)
\end{gathered}
$$

Next, as in regular perturbation methods, we expand the solution $x(\eta, \zeta)$ as a power series in $\epsilon$.

$$
x(\eta, \zeta)=x_{0}(\eta, \zeta)+\epsilon x_{1}(\eta, \zeta)+O\left(\epsilon^{2}\right)
$$

We now substitute (2.3), (2.4), (2.5) into (2.1a) and expand $\omega$. We likewise do this for the $y$ and $w$ oscillators. The resulting equations have terms with coefficients of $O(1), O(\epsilon)$ and higher orders. The procedure now calls for equating the coefficients of each power of $\epsilon$ to zero. The lowest order equations are

$$
\begin{gathered}
\frac{d^{2} x_{0}}{d \zeta^{2}}+x_{0}=0 \\
\frac{d^{2} y_{0}}{d \zeta^{2}}+y_{0}=0 \\
\frac{d^{2} w_{0}}{d \zeta^{2}}+w_{0}=0
\end{gathered}
$$

Each of the $O(1)$ equations represent a harmonic oscillator. We write the solutions to (2.6) in polar form with constants of integration (amplitude and phase) functionally dependent on slow-time, $\eta$.

$$
\begin{aligned}
& x_{0}(\zeta, \eta)=R_{1}(\eta) \cos \left(\zeta-\theta_{1}(\eta)\right) \\
& y_{0}(\zeta, \eta)=R_{2}(\eta) \cos \left(\zeta-\theta_{2}(\eta)\right) \\
& w_{0}(\zeta, \eta)=R_{3}(\eta) \cos \left(\zeta-\theta_{3}(\eta)\right)
\end{aligned}
$$


Next we look at the equations resulting from the $O(\epsilon)$ terms. After plugging in (2.7) and performing some trigonometric simplifications we have

$$
\begin{aligned}
& \frac{d^{2} x_{1}}{d \zeta^{2}}+x_{1}=(\ldots) \cos (\zeta)+(\ldots) \sin (\zeta)+(\ldots) \\
& \frac{d^{2} y_{1}}{d \zeta^{2}}+y_{1}=(\ldots) \cos (\zeta)+(\ldots) \sin (\zeta)+(\ldots) \\
& \frac{d^{2} w_{1}}{d \zeta^{2}}+w_{1}=(\ldots) \cos (\zeta)+(\ldots) \sin (\zeta)+(\ldots)
\end{aligned}
$$

where $(. .$.$) represents constant (independent of \zeta$ ) equations of coefficients involving $R_{1}, R_{2}, R_{3}, \theta_{1}, \theta_{2}, \theta_{3}$ and their respective derivatives (with respect to $\eta$ ). For the slow-flow equations to be uniformly valid for all time we need to eliminate all resonance terms from (2.8) [9]. Thus we set the coefficients of $\cos (\zeta)$ and $\sin (\zeta)$ on the right-hand side of (2.8) equal to zero. This results in six different equations involving the six unknown derivatives $-\frac{d R_{1}}{d \eta}, \frac{d R_{2}}{d \eta}, \frac{d R_{3}}{d \eta}, \frac{d \theta_{1}}{d \eta}, \frac{d \theta_{2}}{d \eta}, \frac{d \theta_{3}}{d \eta}$. Solving this system of six equations and six unknowns yields

$$
\begin{gathered}
\frac{d R_{1}}{d \eta}=-\frac{\mu R_{3}}{2} \sin \left(\theta_{3}-\theta_{1}\right)+\frac{R_{1}}{2}\left(1-\frac{R_{1}^{2}}{4}\right) \\
\frac{d R_{2}}{d \eta}=-\frac{\mu R_{3}}{2} \sin \left(\theta_{3}-\theta_{2}\right)+\frac{R_{2}}{2}\left(1-\frac{R_{2}^{2}}{4}\right) \\
\frac{d R_{3}}{d \eta}=\frac{\mu R_{2}}{2} \sin \left(\theta_{3}-\theta_{2}\right)+\frac{\mu R_{1}}{2} \sin \left(\theta_{3}-\theta_{1}\right)+\frac{R_{3}}{2}\left(1-\frac{R_{3}^{2}}{4}\right) \\
\frac{d \theta_{1}}{d \eta}=\frac{\mu R_{3}}{2 R_{1}} \cos \left(\theta_{3}-\theta_{1}\right)-\frac{\mu}{2}+k_{1} \\
\frac{d \theta_{2}}{d \eta}=\frac{\mu R_{3}}{2 R_{2}} \cos \left(\theta_{3}-\theta_{2}\right)-\frac{\mu}{2}+k_{1} \\
\frac{d \theta_{3}}{d \eta}=\frac{\mu}{2}\left(\frac{R_{2}}{R_{3}} \cos \left(\theta_{3}-\theta_{2}\right)+\frac{R_{1}}{R_{3}} \cos \left(\theta_{3}-\theta_{1}\right)-2\right)+k_{1}-\Delta
\end{gathered}
$$

Equations (2.9) represent the slow-flow for the system (2.1). They describe how the amplitude and phase of the $O(1)$ solutions (2.7) change with respect to slow-time. 
We note that the oscillator phases only appear as differences. If we define the phasedifferences between the $w$ oscillator and the $x$ and $y$ oscillators as $\phi_{1}=\theta_{3}-\theta_{1}$ and $\phi_{2}=\theta_{3}-\theta_{2}$ respectively, we can eliminate one equation from (2.9), thus reducing the dimension of the slow-flow problem by one. With these new definitions the slow-flow equations (2.9) become

$$
\begin{gathered}
\frac{d R_{1}}{d \eta}=-\frac{\mu R_{3}}{2} \sin \left(\phi_{1}\right)+\frac{R_{1}}{2}\left(1-\frac{R_{1}^{2}}{4}\right) \\
\frac{d R_{2}}{d \eta}=-\frac{\mu R_{3}}{2} \sin \left(\phi_{2}\right)+\frac{R_{2}}{2}\left(1-\frac{R_{2}^{2}}{4}\right) \\
\frac{d R_{3}}{d \eta}=\frac{\mu R_{2}}{2} \sin \left(\phi_{2}\right)+\frac{\mu R_{1}}{2} \sin \left(\phi_{1}\right)+\frac{R_{3}}{2}\left(1-\frac{R_{3}^{2}}{4}\right) \\
\frac{d \phi_{1}}{d \eta}=\frac{\mu}{2}\left[\frac{R_{2}}{R_{3}} \cos \left(\phi_{2}\right)+\left(\frac{R_{1}}{R_{3}}-\frac{R_{3}}{R_{1}}\right) \cos \left(\phi_{1}\right)-1\right]-\Delta \\
\frac{d \phi_{2}}{d \eta}=\frac{\mu}{2}\left[\frac{R_{1}}{R_{3}} \cos \left(\phi_{1}\right)+\left(\frac{R_{2}}{R_{3}}-\frac{R_{3}}{R_{2}}\right) \cos \left(\phi_{2}\right)-1\right]-\Delta
\end{gathered}
$$

Note that in finding the slow-flow the term $k_{1}$ was never determined. Thus it was not necessary to include strained time $\zeta$ (as opposed to normal time $t$ ) in our first-order analysis. Later, we use this form of the slow-flow equations to analyze the dynamics of the in-phase mode of (2.1).

\subsection{Slow-Flow in Rectangular Coordinates}

Following the procedure (and using results (2.2) through (2.6)) described in the preceeding section for the two-variable expansion method, we now define the $O(1)$ solutions using rectangular coordinates.

$$
\begin{aligned}
& x_{0}(\zeta, \eta)=A_{1}(\eta) \cos (\zeta)+B_{1}(\eta) \sin (\zeta) \\
& y_{0}(\zeta, \eta)=A_{2}(\eta) \cos (\zeta)+B_{2}(\eta) \sin (\zeta)
\end{aligned}
$$




$$
w_{0}(\zeta, \eta)=A_{3}(\eta) \cos (\zeta)+B_{3}(\eta) \sin (\zeta)
$$

After plugging (2.11) into the $O(\epsilon)$ equations, the elimination of resonance terms now results in the following six rectangular slow-flow equations

$$
\begin{gathered}
\frac{d A_{1}}{d \eta}=\frac{\mu}{2}\left(B_{1}-B_{3}\right)-B_{1} k_{1}-\frac{A_{1}}{2}\left(\frac{B_{1}^{2}}{4}+\frac{A_{1}^{2}}{4}-1\right) \\
\frac{d A_{2}}{d \eta}=\frac{\mu}{2}\left(B_{2}-B_{3}\right)-B_{2} k_{1}-\frac{A_{2}}{2}\left(\frac{B_{2}^{2}}{4}+\frac{A_{2}^{2}}{4}-1\right) \\
\frac{d A_{3}}{d \eta}=\frac{\mu}{2}\left(2 B_{3}-B_{1}-B_{2}\right)-B_{3} k_{1}+B_{3} \Delta-\frac{A_{3}}{2}\left(\frac{B_{3}^{2}}{4}+\frac{A_{3}^{2}}{4}-1\right) \\
\frac{d B_{1}}{d \eta}=\frac{\mu}{2}\left(A_{3}-A_{1}\right)+A_{1} k_{1}-\frac{B_{1}}{2}\left(\frac{B_{1}^{2}}{4}+\frac{A_{1}^{2}}{4}-1\right) \\
\frac{d B_{2}}{d \eta}=\frac{\mu}{2}\left(A_{3}-A_{2}\right)+A_{2} k_{1}-\frac{B_{2}}{2}\left(\frac{B_{2}^{2}}{4}+\frac{A_{2}^{2}}{4}-1\right) \\
\frac{d B_{3}}{d \eta}=\frac{\mu}{2}\left(A_{1}+A_{2}-2 A_{3}\right)+A_{3} k_{1}-A_{2} \Delta-\frac{B_{3}}{2}\left(\frac{B_{3}^{2}}{4}+\frac{A_{3}^{2}}{4}-1\right)
\end{gathered}
$$

We do not use the rectangular form of the slow-flow equations in this thesis, but future work may be aided by an analysis in rectangular coordinates.

\subsection{Slow-Flow via Method of Averaging}

Another popular singular perturbation technique besides two-variable expansion often encountered in the literature is the method of averaging. We now demonstrate the equivalence of these two methods by obtaining the slow-flow equations (2.10) using one-and-a-half order averaging [8].

To use the method of averaging we write the original system of equations (2.1) in the form

$$
\begin{aligned}
& \ddot{x}+x=\epsilon F_{x}(x, \dot{x}, w, t, \mu) \\
& \ddot{y}+y=\epsilon F_{y}(y, \dot{y}, w, t, \mu)
\end{aligned}
$$




$$
\ddot{w}+w=\epsilon F_{w}(w, \dot{w}, x, y, t, \Delta, \mu)
$$

where $F_{x}, F_{y}$, and $F_{w}$ are defined as

$$
\begin{gathered}
F_{x}=\left(1-x^{2}\right) \dot{x}+\mu(w-x) \\
F_{y}=\left(1-y^{2}\right) \dot{y}+\mu(w-y) \\
F_{w}=\left(1-w^{2}\right) \dot{w}+\mu(x+y-2 w)-2 \Delta w
\end{gathered}
$$

When $\epsilon=0$, equations (2.13) define a system of three uncoupled harmonic oscillators, the solutions of which are (in polar form)

$$
\begin{aligned}
& x=R_{1} \cos \left(t-\theta_{1}\right) \\
& y=R_{2} \cos \left(t-\theta_{2}\right) \\
& w=R_{3} \cos \left(t-\theta_{3}\right)
\end{aligned}
$$

with time derivatives

$$
\begin{aligned}
& \dot{x}=-R_{1} \sin \left(t-\theta_{1}\right) \\
& \dot{y}=-R_{2} \sin \left(t-\theta_{2}\right) \\
& \dot{w}=-R_{3} \sin \left(t-\theta_{3}\right)
\end{aligned}
$$

When $\epsilon \neq 0$ we assume the constants of integration in (2.15) and (2.16) to be functions of time and no longer constant. This move is similar to that of the method of variation of parameters when solving linear differential equations [8]. Using the $x$-oscillator as an example, we differentiate (2.15) and require (2.16) to hold.

$$
\dot{x}=\dot{R}_{1} \cos \left(t-\theta_{1}\right)-R_{1}\left(1-\dot{\theta}_{1}\right) \sin \left(t-\theta_{1}\right)=-R_{1} \sin \left(t-\theta_{1}\right)
$$


Next, we differentiate (2.16) and insert it into the original differential equation $(2.13 \mathrm{a})$

$$
\ddot{x}=-\dot{R}_{1} \sin \left(t-\theta_{1}\right)-R_{1}\left(1-\dot{\theta_{1}}\right) \cos \left(t-\theta_{1}\right)=-R_{1} \cos \left(t-\theta_{1}\right)+\epsilon F_{x}
$$

where we have suppressed the dependencies of $F_{x}$. Rearranging (2.17) and (2.18) we get

$$
\begin{gathered}
\dot{R}_{1} \cos \left(t-\theta_{1}\right)+R_{1} \dot{\theta}_{1} \sin \left(t-\theta_{1}\right)=0 \\
-\dot{R}_{1} \sin \left(t-\theta_{1}\right)+R_{1} \dot{\theta}_{1} \cos \left(t-\theta_{1}\right)=\epsilon F_{x}
\end{gathered}
$$

Next we solve (2.19) and (2.20) for $\dot{R}_{1}$ and $\dot{\theta}_{1}$. First we multiply (2.19) by $\cos \left(t-\theta_{1}\right)$ and subtract from it $\sin \left(t-\theta_{1}\right)$ times $(2.20)$ yielding

$$
\dot{R}_{1}=-\epsilon \sin \left(t-\theta_{1}\right) F_{x}
$$

Second, we multiply (2.19) by $\sin \left(t-\theta_{1}\right)$ and add to it $\cos \left(t-\theta_{1}\right)$ times $(2.20)$. Simplifying the resulting equation yields

$$
\dot{\theta_{1}}=-\frac{\epsilon}{R_{1}} \cos \left(t-\theta_{1}\right) F_{x}
$$

Now we posit a near-identity transformation of the form

$$
\begin{aligned}
& R_{1}=\bar{R}_{1}+\epsilon W_{1}\left(\bar{R}_{1}, \overline{\theta_{1}}, \bar{R}_{3}, \overline{\theta_{3}}\right)+O\left(\epsilon^{2}\right) \\
& \theta_{1}=\bar{\theta}_{1}+\epsilon W_{2}\left(\bar{R}_{1}, \bar{\theta}_{1}, \bar{R}_{3}, \bar{\theta}_{3}\right)+O\left(\epsilon^{2}\right)
\end{aligned}
$$

where $W_{1}$ and $W_{2}$ are referred to as the generating functions of the transformation. They will be determined later as to simplify the slow-flow as much as possible. This is the crucial step that differentiates one-and-a-half order averaging from simple averaging [8]. The next step in obtaining the slow-flow equations is to differentiate the near-identity transformations (2.23) with respect to time, yielding

$$
\dot{R_{1}}=\dot{\bar{R}}_{1}+\epsilon\left(\frac{\partial W_{1}}{\partial \bar{R}_{1}} \dot{\dot{R}_{1}}+\frac{\partial W_{1}}{\partial \bar{\theta}_{1}} \dot{\overline{\theta_{1}}}+\frac{\partial W_{1}}{\partial \bar{R}_{3}} \dot{\dot{R}_{3}}+\frac{\partial W_{1}}{\partial \bar{\theta}_{3}} \dot{\dot{\theta}_{3}}+\dot{W}_{1}\right)
$$




$$
\dot{\theta_{1}}=\dot{\overline{\theta_{1}}}+\epsilon\left(\frac{\partial W_{2}}{\partial \bar{R}_{1}} \dot{\overline{R_{1}}}+\frac{\partial W_{2}}{\partial \bar{\theta}_{1}} \dot{\overline{\theta_{1}}}+\frac{\partial W_{2}}{\partial \bar{R}_{3}} \dot{\overline{R_{3}}}+\frac{\partial W_{2}}{\partial \bar{\theta}_{3}} \dot{\bar{\theta}}_{3}+\dot{W}_{2}\right)
$$

We can simplify these expressions by noting that all the time derivatives of barred variables are $O(\epsilon)$ and when multiplied by $\epsilon$ become $O\left(\epsilon^{2}\right)$ and thus can then be safely ignored in our analysis. Equations (2.24a) and (2.24b) then reduce to

$$
\begin{gathered}
\dot{R}_{1}=\dot{\bar{R}}_{1}+\epsilon\left(\frac{\partial W_{1}}{\partial t}\right) \\
\dot{\theta_{1}}=\dot{\bar{\theta}_{1}}+\epsilon\left(\frac{\partial W_{2}}{\partial t}\right)
\end{gathered}
$$

To find the differential equations governing the new transformed variables we rewrite (2.25) by plugging in (2.21) and (2.22) for $\dot{R}_{1}$ and $\dot{\theta}_{1}$ respectively and solving for $\dot{\bar{R}}_{1}$ and $\dot{\overline{\theta_{1}}}$, giving

$$
\begin{gathered}
\dot{\overline{R_{1}}}=-\epsilon\left(\sin \left(t-\overline{\theta_{1}}\right) F_{x}+\frac{\partial W_{1}}{\partial t}\right) \\
\dot{\overline{\theta_{1}}}=\epsilon\left(\frac{1}{\bar{R}_{1}} \cos \left(t-\overline{\theta_{1}}\right) F_{x}-\frac{\partial W_{2}}{\partial t}\right)
\end{gathered}
$$

The final step of the averaging method is to pick the generating functions $W_{1}$ and $W_{2}$ to simplify (2.26) and (2.27) as much as possible. In particular, we select $W_{1}$ and $W_{2}$ to eliminate all trigonometric functions depending on time explicitly. After this substitution and subsequent algebraic manipulation, this yields the following differential equations for the transformed variables

$$
\begin{gathered}
\dot{\dot{R}_{1}}=-\epsilon\left(\frac{\mu}{2} \bar{R}_{3} \sin \left(\overline{\theta_{3}}-\overline{\theta_{1}}\right)+\frac{\bar{R}_{1}^{3}}{8}-\frac{\bar{R}_{1}}{2}\right) \\
\dot{\overline{\theta_{1}}}=\epsilon\left(\frac{\mu \bar{R}_{3}}{2 \bar{R}_{1}} \cos \left(\overline{\theta_{3}}-\overline{\theta_{1}}\right)-\frac{\mu}{2}\right)
\end{gathered}
$$

These equations can be made equivalent to the slow-flow equations (2.9a) and (2.9d) found earlier via two-variable expansion by once again defining a new slowtime variable (see 2.2 ), and writing $\bar{R}_{1}$ and $\overline{\theta_{1}}$ as functions of this new time variable.

$$
\dot{\bar{R}_{1}}=\frac{d \bar{R}_{1}}{d \eta} \dot{\eta}=\epsilon \frac{d \bar{R}_{1}}{d \eta}
$$




$$
\dot{\overline{\theta_{1}}}=\frac{d \overline{\theta_{1}}}{d \eta} \dot{\eta}=\epsilon \frac{d \overline{\theta_{1}}}{d \eta}
$$

Then by dividing (2.28) and (2.29) through by $\epsilon$ we recover the slow-flow equations $(2.9 \mathrm{a})$ and $(2.9 \mathrm{~d})\left(\right.$ with $\left.k_{1}=0\right)$.

$$
\begin{gathered}
\frac{d \bar{R}_{1}}{d \eta}=-\frac{\mu \bar{R}_{3}}{2} \sin \left(\overline{\theta_{3}}-\overline{\theta_{1}}\right)+\frac{\bar{R}_{1}}{2}\left(1-\frac{\bar{R}_{1}^{2}}{4}\right) \\
\frac{d \overline{\theta_{1}}}{d \eta}=\frac{\mu \bar{R}_{3}}{2 \bar{R}_{1}} \cos \left(\overline{\theta_{3}}-\overline{\theta_{1}}\right)-\frac{\mu}{2}
\end{gathered}
$$

In the same manner we can find equivalent slow-flow equations for $\bar{R}_{2}, \bar{R}_{3}, \bar{\theta}_{2}$, and $\overline{\theta_{3}}$ that match those obtained earlier via two-variable expansion. 
CHAPTER 3

\section{INVESTIGATION OF THE PARAMETER SPACE FOR THE IN-PHASE MODE}

The in-phase mode $(x=y)$ resides in a 4-dimensional subspace of the original problem (2.1) and satisfies

$$
\begin{gathered}
\ddot{x}-\epsilon\left(1-x^{2}\right) \dot{x}+x=\epsilon \mu(w-x) \\
\ddot{w}-\epsilon\left(1-w^{2}\right) \dot{w}+p^{2} w=\epsilon 2 \mu(x-w)
\end{gathered}
$$

The slow-flow equations for the in-phase mode are found easily by setting $R_{1}=R_{2}$ and $\phi_{1}=\phi_{2}$ in (2.10). With these substitutions the slow-flow for the in-phase mode, ignoring redundant equations, is

$$
\begin{gathered}
\frac{d R_{1}}{d \eta}=-\frac{\mu R_{3}}{2} \sin \phi+\frac{R_{1}}{2}\left(1-\frac{R_{1}^{2}}{4}\right) \\
\frac{d R_{3}}{d \eta}=\mu R_{1} \sin \phi+\frac{R_{3}}{2}\left(1-\frac{R_{3}^{2}}{4}\right) \\
\frac{d \phi}{d \eta}=\frac{\mu}{2}\left[\left(\frac{2 R_{1}}{R_{3}}-\frac{R_{3}}{R_{1}}\right) \cos \phi-1\right]-\Delta
\end{gathered}
$$

The above set of equations represent a three-dimensional invariant subspace of the

original slow-flow (2.10). Mathematically, the subspace is defined as the Cartesian product of $R^{+} \times R^{+} \times S^{1}$.

To find equilibrium points of the in-phase slow-flow we set equations (3.3) equal to 0 .

$$
\begin{gathered}
-\frac{\mu R_{3}}{2} \sin \phi+\frac{R_{1}}{2}\left(1-\frac{R_{1}^{2}}{4}\right)=0 \\
\mu R_{1} \sin \phi+\frac{R_{3}}{2}\left(1-\frac{R_{3}^{2}}{4}\right)=0
\end{gathered}
$$




$$
\frac{\mu}{2}\left[\left(\frac{2 R_{1}}{R_{3}}-\frac{R_{3}}{R_{1}}\right) \cos \phi-1\right]-\Delta=0
$$

Thus, for a particular pair of $\mu$ and $\Delta$ in parameter space, an equilibrium point must satisfy all three of these nonlinear equations.

\subsection{Saddle-Node Bifurcation Curves}

To find the curves in parameter space representing saddle-node bifurcations (or folds) of equilibria we first need to manipulate the three equilibrium equations (3.4) into a more usable form. We now follow essentially the procedure given in [4], [9] for the problem of two weakly-coupled van der Pol oscillators.

First we solve equation (3.4c) for $\cos (\phi)$, giving

$$
\cos (\phi)=\frac{-(\mu+2 \Delta) R_{1} R_{3}}{\mu\left(R_{3}^{2}-2 R_{1}^{2}\right)}
$$

Next we find two linearly independent linear combinations of the remaining two

equations. First, we multiply (3.4b) by $R_{1}$ and subtract from it $R_{3}$ times (3.4a) and solve for $\sin (\phi)$.

$$
\sin (\phi)=\frac{R_{1} R_{3}\left(R_{3}^{2}-R_{1}^{2}\right)}{4 \mu\left(R_{3}^{2}+2 R_{1}^{2}\right)}
$$

We can now use the identity $\cos ^{2} \phi+\sin ^{2} \phi=1$ to get

$$
\frac{R_{1}^{2} R_{3}^{2}(\mu+2 \Delta)^{2}}{\mu^{2}\left(R_{3}^{2}-2 R_{1}^{2}\right)^{2}}+\frac{R_{1}^{2} R_{3}^{2}\left(R_{3}^{2}-R_{1}^{2}\right)^{2}}{16 \mu^{2}\left(R_{3}^{2}+2 R_{1}^{2}\right)^{2}}=1
$$

This gives us the first of our independent equations. We find a second one by multiplying (3.4a) by $2 R_{1}$ and adding to it $R_{3}$ times (3.4b) to get

$$
R_{3}^{2}+2 R_{1}^{2}=\frac{1}{4}\left(R_{3}^{4}+2 R_{1}^{4}\right)
$$


Equations (3.7) and (3.8) allow us to find possible saddle-node bifurcation curves in the $\Delta-\mu$ parameter space. We first eliminate $R_{3}$ from both equations. The resulting equation (neglecting any unnecessary factors) is further simplified by replacing $R_{1}$ with $\bar{R}_{1}=R_{1}^{2}$ giving us

$$
\begin{aligned}
& 9 \bar{R}_{1}{ }^{6}-156 \bar{R}_{1}{ }^{5}-32 \mu^{2} \bar{R}_{1}^{4}-128 \Delta \mu \bar{R}_{1}^{4}-128 \Delta^{2} \bar{R}_{1}^{4}+1072 \bar{R}_{1}^{4}-576 \mu^{2} \bar{R}_{1}^{3} \\
& -3648 \bar{R}_{1}{ }^{3}+6912 \mu^{4} \bar{R}_{1}{ }^{2}+10240 \Delta \mu^{3} \bar{R}_{1}{ }^{2}+14336 \Delta^{2} \mu^{2} \bar{R}_{1}{ }^{2}+7168 \mu^{2} \bar{R}_{1}{ }^{2} \\
& +8192 \Delta^{3} \mu \bar{R}_{1}{ }^{2}+7168 \Delta \mu \bar{R}_{1}{ }^{2}+4096 \Delta^{4} \bar{R}_{1}{ }^{2}+7168 \Delta^{2} \bar{R}_{1}{ }^{2}+6144 \bar{R}_{1}{ }^{2} \\
& -36864 \mu^{4} \bar{R}_{1}-53248 \Delta \mu^{3} \bar{R}_{1}-69632 \Delta^{2} \mu^{2} \bar{R}_{1}-21504 \mu^{2} \bar{R}_{1}-32768 \Delta^{3} \mu \bar{R}_{1} \\
& -20480 \Delta \mu \bar{R}_{1}-16384 \Delta^{4} \bar{R}_{1}-20480 \Delta^{2} \bar{R}_{1}-4096 \bar{R}_{1}+36864 \mu^{4}+16384 \Delta \mu^{3} \\
& +16384 \Delta^{2} \mu^{2}+16384 \mu^{2}=0
\end{aligned}
$$

Since a saddle-node bifurcation will occur at a double root, we further require that the derivative of (3.9) with respect to $\bar{R}_{1}$ also be satisfied.

$$
\begin{aligned}
& 54 \bar{R}_{1}{ }^{5}-780 \bar{R}_{1}{ }^{4}-128 \mu^{2} \bar{R}_{1}{ }^{3}-512 \Delta \mu \bar{R}_{1}{ }^{3}-512 \Delta^{2} \bar{R}_{1}{ }^{3}+4288 \bar{R}_{1}{ }^{3}-1728 \mu^{2} \bar{R}_{1}{ }^{2} \\
& -10944 \bar{R}_{1}{ }^{2}+13824 \mu^{4} \bar{R}_{1}+20480 \Delta \mu^{3} \bar{R}_{1}+28672 \Delta^{2} \mu^{2} \bar{R}_{1}+14336 \mu^{2} \bar{R}_{1} \\
& +16384 \Delta^{3} \mu \bar{R}_{1}+14336 \Delta \mu \bar{R}_{1}+8192 \Delta^{4} \bar{R}_{1}+14336 \Delta^{2} \bar{R}_{1}+12288 \bar{R}_{1}-36864 \mu^{4} \\
& -53248 \Delta \mu^{3}-69632 \Delta^{2} \mu^{2}-21504 \mu^{2}-32768 \Delta^{3} \mu-20480 \Delta \mu-16384 \Delta^{4} \\
& -20480 \Delta^{2}-4096=0
\end{aligned}
$$

We can now eliminate $\bar{R}_{1}$ from (3.9) and (3.10) to obtain an equation in terms of $\Delta$ and $\mu$ only. Using MACSYMA, this results in three different curves. The first 
curve is given by

$$
\begin{aligned}
& 45349632 \mu^{14}+221709312 \Delta \mu^{13}+690508800 \Delta^{2} \mu^{12}+9027936 \mu^{12} \\
& +492475904 \Delta^{3} \mu^{11}+38320128 \Delta \mu^{11}+2529857536 \Delta^{4} \mu^{10}+105827328 \Delta^{2} \mu^{10} \\
& -4323051 \mu^{10}+3420995584 \Delta^{5} \mu^{9}+231647232 \Delta^{3} \mu^{9}-5105708 \Delta \mu^{9} \\
& +3827613696 \Delta^{6} \mu^{8}+453522432 \Delta^{4} \mu^{8}-35909484 \Delta^{2} \mu^{8}+539217 \mu^{8} \\
& +3541827584 \Delta^{7} \mu^{7}+721944576 \Delta^{5} \mu^{7}-63304320 \Delta^{3} \mu^{7}+3036832 \Delta \mu^{7} \\
& +2745761792 \Delta^{8} \mu^{6}+919879680 \Delta^{6} \mu^{6}-80637888 \Delta^{4} \mu^{6}+8802064 \Delta^{2} \mu^{6} \\
& -12636 \mu^{6}+1759248384 \Delta^{9} \mu^{5}+912162816 \Delta^{7} \mu^{5}-38032128 \Delta^{5} \mu^{5} \\
& +7835616 \Delta^{3} \mu^{5}-183192 \Delta \mu^{5}+930873344 \Delta^{10} \mu^{4}+701669376 \Delta^{8} \mu^{4} \\
& +40007424 \Delta^{6} \mu^{4}-3381904 \Delta^{4} \mu^{4}-841176 \Delta^{2} \mu^{4}-81 \mu^{4}+392167424 \Delta^{11} \mu^{3} \\
& +410910720 \Delta^{9} \mu^{3}+81358848 \Delta^{7} \mu^{3}-3334912 \Delta^{5} \mu^{3}-1019520 \Delta^{3} \mu^{3} \\
& +2640 \Delta \mu^{3}+128974848 \Delta^{12} \mu^{2}+177340416 \Delta^{10} \mu^{2}+65590272 \Delta^{8} \mu^{2} \\
& +7929600 \Delta^{6} \mu^{2}+231360 \Delta^{4} \mu^{2}+20304 \Delta^{2} \mu^{2}+29360128 \Delta^{13} \mu+51904512 \Delta^{11} \mu \\
& +30167040 \Delta^{9} \mu+7749632 \Delta^{7} \mu+889344 \Delta^{5} \mu+35328 \Delta^{3} \mu+16 \Delta \mu \\
& +4194304 \Delta^{14}+8650752 \Delta^{12}+6033408 \Delta^{10}+1937408 \Delta^{8}+296448 \Delta^{6} \\
& +17664 \Delta^{4}+16 \Delta^{2}=0
\end{aligned}
$$

Equation (3.11) plots as two triangular regions in parameter space (see Figure 3.1). The sides of the triangular regions represent either a saddle-node or an infiniteperiod bifurcation of equilibria (see Chapter 4).

The second saddle-node bifurcation curve equation found is

$$
\mu+2 \Delta=0
$$

Equation (3.12) is a straight line bisecting the triangular regions given by (3.11) 


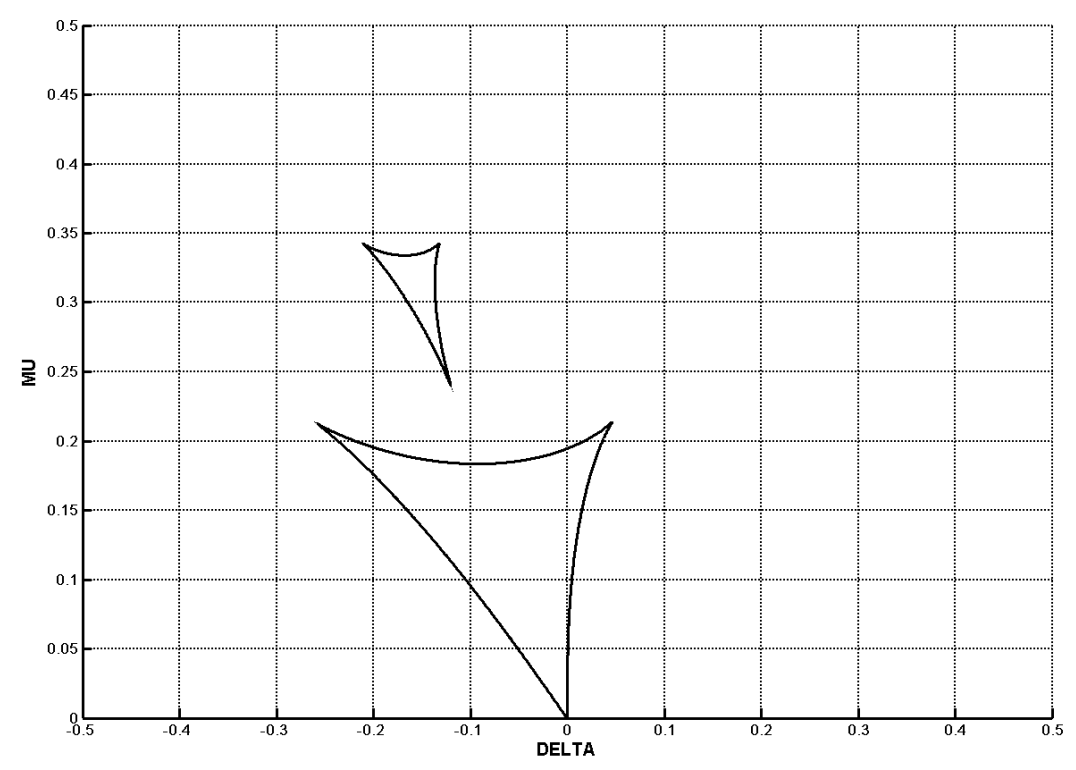

Figure 3.1: Saddle-node bifurcation curves in parameter space.

and does not correspond to bifurcations taking place in the 3-dimensional in-phase slow-flow subspace. However, equation (3.12) does appear to be a bifurcation curve in the larger 5-dimensional slow-flow (2.10) and thus will play a roll when studying the larger 5-dimensional problem. A third equation is also obtained but it does not represent a bifurcation curve of the in-phase slow-flow equations and has no significance to this problem. It is due to additional zeros added to the problem by MACSYMA in the course of eliminating variables. These extra zeros appear every time the ELIMINATE command is used and so it is understood from now on we simply ignore these extraneous zeros. 


\subsection{Hopf Bifurcation Curves}

Next we seek to find conditions for the existence of any limits cycles present in the in-phase slow-flow. They will be born in a supercritical Hopf bifurcation where a stable equilibria loses its stability when two of its eigenvalues become purely imaginary. Let $\left(\bar{R}_{1}, \bar{R}_{3}, \bar{\phi}\right)$ be such an equilibrium point (satisfying (3.4)). To investigate the behavior of its eigenvalues we linearize the in-phase slow-flow (3.4) around the equilibrium point, resulting in the Jacobian matrix

$$
D f=\left[\begin{array}{ccc}
\frac{1}{3}-\frac{3 \bar{R}_{1}{ }^{2}}{8} & -\frac{\mu \sin \bar{\phi}}{2} & -\frac{\mu \bar{R}_{3} \cos \bar{\phi}}{2} \\
\mu \sin \bar{\phi} & \frac{1}{3}-\frac{3 \bar{R}_{3}{ }^{2}}{8} & \mu \bar{R}_{1} \cos \phi \\
\frac{\mu \bar{R}_{3} \cos \bar{\phi}}{2 \bar{R}_{1}{ }^{2}}+\frac{\mu \cos \bar{\phi}}{R_{3}} & -\frac{\mu \bar{R}_{1} \cos \bar{\phi}}{\bar{R}_{3}{ }^{2}}-\frac{\mu \cos \bar{\phi}}{2 R_{1}} & \frac{\mu \bar{R}_{3} \sin \bar{\phi}}{2 R_{1}}-\frac{\mu \bar{R}_{1} \sin \bar{\phi}}{R_{3}}
\end{array}\right]
$$

The Jacobian can be simplified by using equations (3.5) and (3.6) to eliminate $\cos (\phi)$ and $\sin (\phi)$. The resulting characteristic equation, which contains only $\bar{R}_{1}$ and $\bar{R}_{3}$ is of the form

$$
\lambda^{3}+c_{2} \lambda^{2}+c_{1} \lambda+c_{0}=0
$$

where the coefficients $c_{0}, c_{1}, c_{2}$ are given as

$$
\begin{gathered}
c_{2}=-\frac{\bar{R}_{3}{ }^{4}+6{\overline{R_{1}}}^{2} \bar{R}_{3}{ }^{2}-4 \bar{R}_{3}{ }^{2}+2 \bar{R}_{1}{ }^{4}-8 \bar{R}_{1}{ }^{2}}{4\left(\bar{R}_{3}{ }^{2}+2 \bar{R}_{1}{ }^{2}\right)} \\
c_{1}=\left(3 \bar{R}_{3}{ }^{12}-23 \bar{R}_{1}{ }^{2} \bar{R}_{3}{ }^{10}+4 \bar{R}_{3}{ }^{10}+9 \bar{R}_{1}{ }^{4} \bar{R}_{3}{ }^{8}+52 \bar{R}_{1}{ }^{2} \bar{R}_{3}{ }^{8}-16 \mu^{2} \bar{R}_{3}{ }^{8}-64 \Delta \mu \bar{R}_{3}{ }^{8}\right. \\
-64 \Delta^{2} \bar{R}_{3}{ }^{8}-16 \bar{R}_{3}{ }^{8}+106 \bar{R}_{1}{ }^{6} \bar{R}_{3}{ }^{6}-128 \bar{R}_{1}{ }^{4} \bar{R}_{3}{ }^{6}-128 \mu^{2} \bar{R}_{1}{ }^{2} \bar{R}_{3}{ }^{6}-512 \Delta \mu \bar{R}_{1}{ }^{2} \bar{R}_{3}{ }^{6} \\
-512 \Delta^{2} \bar{R}_{1}{ }^{2} \bar{R}_{3}{ }^{6}-24 \bar{R}_{1}{ }^{8} \bar{R}_{3}{ }^{4}-224 \bar{R}_{1}{ }^{6} \bar{R}_{3}{ }^{4}-384 \mu^{2} \bar{R}_{1}{ }^{4} \bar{R}_{3}{ }^{4}-1536 \Delta \mu \bar{R}_{1}{ }^{4} \bar{R}_{3}{ }^{4} \\
-1536 \Delta^{2} \bar{R}_{1}{ }^{4} \bar{R}_{3}{ }^{4}+128 \bar{R}_{1}{ }^{4} \bar{R}_{3}{ }^{4}-200 \bar{R}_{1}{ }^{10} \bar{R}_{3}{ }^{2}+448 \bar{R}_{1}{ }^{8} \bar{R}_{3}{ }^{2}-512 \mu^{2} \bar{R}_{1}{ }^{6} \bar{R}_{3}{ }^{2} \\
-2048 \Delta \mu \bar{R}_{1}{ }^{6} \bar{R}_{3}{ }^{2}-2048 \Delta^{2} \bar{R}_{1}{ }^{6} \bar{R}_{3}{ }^{2}+48 \bar{R}_{1}{ }^{12}+64 \bar{R}_{1}{ }^{10}-256 \mu^{2} \bar{R}_{1}{ }^{8}-1024 \Delta \mu \bar{R}_{1}{ }^{8} \\
\left.-1024 \Delta^{2} \bar{R}_{1}{ }^{8}-256 \bar{R}_{1}{ }^{8}\right) /\left(64\left(\bar{R}_{3}{ }^{2}-2 \bar{R}_{1}{ }^{2}\right)^{2}\left(\bar{R}_{3}{ }^{2}+2 \bar{R}_{1}{ }^{2}{ }^{2}\right)\right.
\end{gathered}
$$




$$
\begin{aligned}
& c_{0}=-\left(\left(11 \bar{R}_{1}{ }^{2} \bar{R}_{3}{ }^{14}-12 \bar{R}_{3}{ }^{14}-45 \bar{R}_{1}^{4} \bar{R}_{3}{ }^{12}+24 \bar{R}_{1}{ }^{2} \bar{R}_{3}{ }^{12}-48 \mu^{2} \bar{R}_{3}{ }^{12}\right.\right. \\
& -192 \Delta \mu \bar{R}_{3}{ }^{12}-192 \Delta^{2} \bar{R}_{3}{ }^{12}+16 \bar{R}_{3}{ }^{12}+12 \bar{R}_{1}{ }^{6} \bar{R}_{3}{ }^{10}+108 \bar{R}_{1}{ }^{4} \bar{R}_{3}{ }^{10}-384 \mu^{2} \bar{R}_{1}{ }^{2} \bar{R}_{3}{ }^{10} \\
& -1536 \Delta \mu{\overline{R_{1}}}^{2} \bar{R}_{3}{ }^{10}-1536 \Delta^{2} \bar{R}_{1}{ }^{2} \bar{R}_{3}{ }^{10}-48 \bar{R}_{1}{ }^{2} \bar{R}_{3}{ }^{10}+64 \mu^{2} \bar{R}_{3}{ }^{10}+256 \Delta \mu \bar{R}_{3}{ }^{10} \\
& +256 \Delta^{2} \bar{R}_{3}{ }^{10}+90 \bar{R}_{1}{ }^{8} \bar{R}_{3}{ }^{8}-216 \bar{R}_{1}{ }^{6} \bar{R}_{3}{ }^{8}-1248 \mu^{2} \bar{R}_{1}{ }^{4} \bar{R}_{3}{ }^{8}-4992 \Delta \mu \bar{R}_{1}{ }^{4} \bar{R}_{3}{ }^{8} \\
& -4992 \Delta^{2} \bar{R}_{1}{ }^{4} \bar{R}_{3}{ }^{8}-96{\overline{R_{1}}}^{4} \bar{R}_{3}{ }^{8}+640 \mu^{2}{\overline{R_{1}}}^{2} \bar{R}_{3}{ }^{8}+2560 \Delta \mu{\overline{R_{1}}}^{2}{\overline{R_{3}}}^{8}+2560 \Delta^{2}{\overline{R_{1}}}^{2}{\overline{R_{3}}}^{8} \\
& +132 \bar{R}_{1}{ }^{10} \bar{R}_{3}{ }^{6}-288 \bar{R}_{1}{ }^{8} \bar{R}_{3}{ }^{6}-2304 \mu^{2} \bar{R}_{1}{ }^{6} \bar{R}_{3}{ }^{6}-9216 \Delta \mu \bar{R}_{1}{ }^{6} \bar{R}_{3}{ }^{6}-9216 \Delta^{2} \bar{R}_{1}{ }^{6} \bar{R}_{3}{ }^{6} \\
& +384 \bar{R}_{1}{ }^{6} \bar{R}_{3}{ }^{6}+2560 \mu^{2} \bar{R}_{1}{ }^{4} \bar{R}_{3}{ }^{6}+10240 \Delta \mu \bar{R}_{1}{ }^{4} \bar{R}_{3}{ }^{6}+10240 \Delta^{2} \bar{R}_{1}{ }^{4} \bar{R}_{3}{ }^{6} \\
& -504 \bar{R}_{1}{ }^{12} \bar{R}_{3}{ }^{4}+576 \bar{R}_{1}{ }^{10} \bar{R}_{3}^{4}-3072 \mu^{2} \bar{R}_{1}{ }^{8} \bar{R}_{3}^{4}-12288 \Delta \mu \bar{R}_{1}{ }^{8} \bar{R}_{3}^{4} \\
& -12288 \Delta^{2} \bar{R}_{1}{ }^{8} \bar{R}_{3}{ }^{4}+5120 \mu^{2} \bar{R}_{1}{ }^{6} \bar{R}_{3}{ }^{4}+20480 \Delta \mu \bar{R}_{1}{ }^{6} \bar{R}_{3}{ }^{4}+20480 \Delta^{2} \bar{R}_{1}{ }^{6} \bar{R}_{3}{ }^{4} \\
& +304 \bar{R}_{1}{ }^{14}{\overline{R_{3}}}^{2}+192 \bar{R}_{1}{ }^{12}{\overline{R_{3}}}^{2}-3072 \mu^{2}{\overline{R_{1}}}^{10}{\overline{R_{3}}}^{2}-12288 \Delta \mu \bar{R}_{1}{ }^{10} \bar{R}_{3}{ }^{2} \\
& -12288 \Delta^{2} \bar{R}_{1}{ }^{10}{\overline{R_{3}}}^{2}-768 \bar{R}_{1}{ }^{10} \bar{R}_{3}{ }^{2}+5120 \mu^{2} \bar{R}_{1}{ }^{8} \bar{R}_{3}{ }^{2}+20480 \Delta \mu{\overline{R_{1}}}^{8} \bar{R}_{3}{ }^{2} \\
& +20480 \Delta^{2} \bar{R}_{1}^{8} \bar{R}_{3}{ }^{2}-384 \bar{R}_{1}{ }^{14}-1536 \mu^{2} \bar{R}_{1}{ }^{12}-6144 \Delta \mu \bar{R}_{1}{ }^{12}-6144 \Delta^{2} \bar{R}_{1}{ }^{12} \\
& \left.+512 \bar{R}_{1}{ }^{12}+2048 \mu^{2} \bar{R}_{1}{ }^{10}+8192 \Delta \mu \bar{R}_{1}{ }^{10}+8192 \Delta^{2} \bar{R}_{1}{ }^{10}\right) /\left(5 1 2 ( { \overline { R _ { 3 } } } ^ { 2 } - 2 { \overline { R _ { 1 } } } ^ { 2 } ) ^ { 2 } \left(\bar{R}_{3}{ }^{2}\right.\right. \\
& \left.\left.\left.+2 \bar{R}_{1}^{2}\right)^{3}\right)\right)
\end{aligned}
$$

For a Hopf bifurcation to occur, there must exist a pair of purely imaginary eigenvalues satisfying (3.14). This requires the characteristic equation to be of the form $[9]$

$$
\lambda^{3}-\alpha \lambda^{2}+\beta^{2} \lambda-\beta^{2} \alpha=0
$$

Equation (3.16) requires that

$$
c_{0}=c_{1} c_{2}
$$

To turn (3.17) into an equation representing a curve in parameter space, we have to eliminate $\bar{R}_{1}$ and $\bar{R}_{3}$ using equations (3.8) and (3.9). Using (3.8) to eliminate 
$\bar{R}_{3}$ we can reduce (3.17) to

$$
\begin{aligned}
& 8019 \overline{\bar{R}}_{1}^{8}-138672 \bar{R}_{1}^{7}+11232 \mu^{2} \bar{R}_{1}^{6}+44928 \Delta \mu \overline{\bar{R}}_{1}^{6}+44928 \Delta^{2} \overline{\bar{R}}_{1}^{6}+963468 \overline{\bar{R}}_{1}^{6} \\
& -101376 \mu^{2} \overline{\bar{R}}_{1}^{5}-405504 \Delta \mu \bar{R}_{1}^{5}-405504 \Delta^{2} \overline{\bar{R}}_{1}^{5}-3380832 \bar{R}_{1}^{5}+6912 \mu^{4} \overline{\bar{R}}_{1}^{4} \\
& +55296 \Delta \mu^{3} \overline{\bar{R}}_{1}^{4}+165888 \Delta^{2} \mu^{2} \overline{\bar{R}}_{1}^{4}+195456 \mu^{2} \overline{\bar{R}}_{1}^{4}+221184 \Delta^{3} \mu \overline{\bar{R}}_{1}^{4}+781824 \Delta \mu \bar{R}_{1}^{4} \\
& +110592 \Delta^{4} \overline{\bar{R}}_{1}^{4}+781824 \Delta^{2} \overline{\bar{R}}_{1}^{4}+6014784 \overline{\bar{R}}_{1}^{4}-36864 \mu^{4} \overline{\bar{R}}_{1}^{3}-294912 \Delta \mu^{3} \overline{\bar{R}}_{1}^{3} \\
& -884736 \Delta^{2} \mu^{2} \overline{\bar{R}}_{1}^{3}+455168 \mu^{2} \overline{\bar{R}}_{1}^{3}-1179648 \Delta^{3} \mu \overline{\bar{R}}_{1}^{3}+1820672 \Delta \mu \overline{\bar{R}_{1}^{3}} \\
& -589824 \Delta^{4} \overline{\bar{R}}_{1}^{3}+1820672 \Delta^{2} \overline{\bar{R}}_{1}^{3}-4143616 \overline{\bar{R}}_{1}^{3}+27648 \mu^{4} \overline{\bar{R}}_{1}^{2}+221184 \Delta \mu^{3} \overline{\bar{R}}_{1}^{2} \\
& +663552 \Delta^{2} \mu^{2} \overline{\bar{R}}_{1}^{2}-1459200 \mu^{2} \overline{\bar{R}}_{1}^{2}+884736 \Delta^{3} \mu \overline{\bar{R}}_{1}^{2}-5836800 \Delta \mu \overline{\bar{R}}_{1}^{2} \\
& +442368 \Delta^{4} \overline{\bar{R}}_{1}^{2}-5836800 \Delta^{2} \overline{\bar{R}}_{1}^{2}-1290240 \bar{R}_{1}^{2}+294912 \mu^{2} \overline{\bar{R}}_{1}+1179648 \Delta \mu \overline{\bar{R}}_{1} \\
& +1179648 \Delta^{2} \overline{\bar{R}}_{1}+1867776 \overline{\bar{R}}_{1}+524288 \mu^{2}+2097152 \Delta \mu+2097152 \Delta^{2} \\
& +524288=0
\end{aligned}
$$

where $\overline{\bar{R}}_{1}=\bar{R}_{1}^{2}$. Finally, we use (3.9) to eliminate $\overline{\bar{R}}_{1}$, giving us

$$
\begin{aligned}
& 206046997776 \mu^{16}+128246239872 \Delta \mu^{15}-1055299653504 \Delta^{2} \mu^{14} \\
& +151716144096 \mu^{14}-4792910330880 \Delta^{3} \mu^{13}-959470912224 \Delta \mu^{13} \\
& -10067384941056 \Delta^{4} \mu^{12}-4022175416544 \Delta^{2} \mu^{12}-76183604811 \mu^{12} \\
& -13620666378240 \Delta^{5} \mu^{11}-8422624949760 \Delta^{3} \mu^{11}-1162076374872 \Delta \mu^{11} \\
& -11038023456768 \Delta^{6} \mu^{10}-9771098692608 \Delta^{4} \mu^{10}-2453769115848 \Delta^{2} \mu^{10} \\
& -53727633963 \mu^{10}-1633265565696 \Delta^{7} \mu^{9}-5111334563328 \Delta^{5} \mu^{9} \\
& -2698414682336 \Delta^{3} \mu^{9}-368730619308 \Delta \mu^{9}+10450239639552 \Delta^{8} \mu^{8} \\
& +4596633773568 \Delta^{6} \mu^{8}-930295796592 \Delta^{4} \mu^{8}-500042378940 \Delta^{2} \mu^{8}-\ldots
\end{aligned}
$$




$$
\begin{aligned}
& \ldots-10567871928 \mu^{8}+18955313086464 \Delta^{9} \mu^{7}+14654584700928 \Delta^{7} \mu^{7} \\
& +2928072365568 \Delta^{5} \mu^{7}-65251243872 \Delta^{3} \mu^{7}-43666800936 \Delta \mu^{7} \\
& +20325924864000 \Delta^{10} \mu^{6}+19734795325440 \Delta^{8} \mu^{6}+6459457683968 \Delta^{6} \mu^{6} \\
& +646749530928 \Delta^{4} \mu^{6}-25863482760 \Delta^{2} \mu^{6}-842897393 \mu^{6} \\
& +15864307384320 \Delta^{11} \mu^{5}+18526299439104 \Delta^{9} \mu^{5}+7894980157440 \Delta^{7} \mu^{5} \\
& +1385895931968 \Delta^{5} \mu^{5}+75604570176 \Delta^{3} \mu^{5}-1221626868 \Delta \mu^{5} \\
& +9417278619648 \Delta^{12} \mu^{4}+12970012852224 \Delta^{10} \mu^{4}+6670762715136 \Delta^{8} \mu^{4} \\
& +1563045302976 \Delta^{6} \mu^{4}+151762831008 \Delta^{4} \mu^{4}+2639537484 \Delta^{2} \mu^{4} \\
& -16895076 \mu^{4}+4277139406848 \Delta^{13} \mu^{3}+6902205382656 \Delta^{11} \mu^{3} \\
& +4225522688000 \Delta^{9} \mu^{3}+1243790340096 \Delta^{7} \mu^{3}+175856646912 \Delta^{5} \mu^{3} \\
& +9882465920 \Delta^{3} \mu^{3}+104063568 \Delta \mu^{3}+1433187385344 \Delta^{14} \mu^{2} \\
& +2690954035200 \Delta^{12} \mu^{2}+1939282108416 \Delta^{10} \mu^{2}+685956946944 \Delta^{8} \mu^{2} \\
& +123792201984 \Delta^{6} \mu^{2}+10341576000 \Delta^{4} \mu^{2}+273469584 \Delta^{2} \mu^{2} \\
& +1086528 \mu^{2}+328866988032 \Delta^{15} \mu+711039909888 \Delta^{13} \mu \\
& +596824129536 \Delta^{11} \mu+250006241280 \Delta^{9} \mu+55862845440 \Delta^{7} \mu \\
& +6480411648 \Delta^{5} \mu+338812032 \Delta^{3} \mu+5262144 \Delta \mu \\
& +41108373504 \Delta^{16}+101577129984 \Delta^{14}+99470688256 \Delta^{12} \\
& +50001248256 \Delta^{10}+13965711360 \Delta^{8}+2160137216 \Delta^{6} \\
& +169406016 \Delta^{4}+5262144 \Delta^{2}+43264=0
\end{aligned}
$$

Equation (3.19) is plotted in Figure 3.2. The dashed lines represent sections of the curve where condition (3.16) no longer hold and thus no Hopf bifurcation occurs there. 


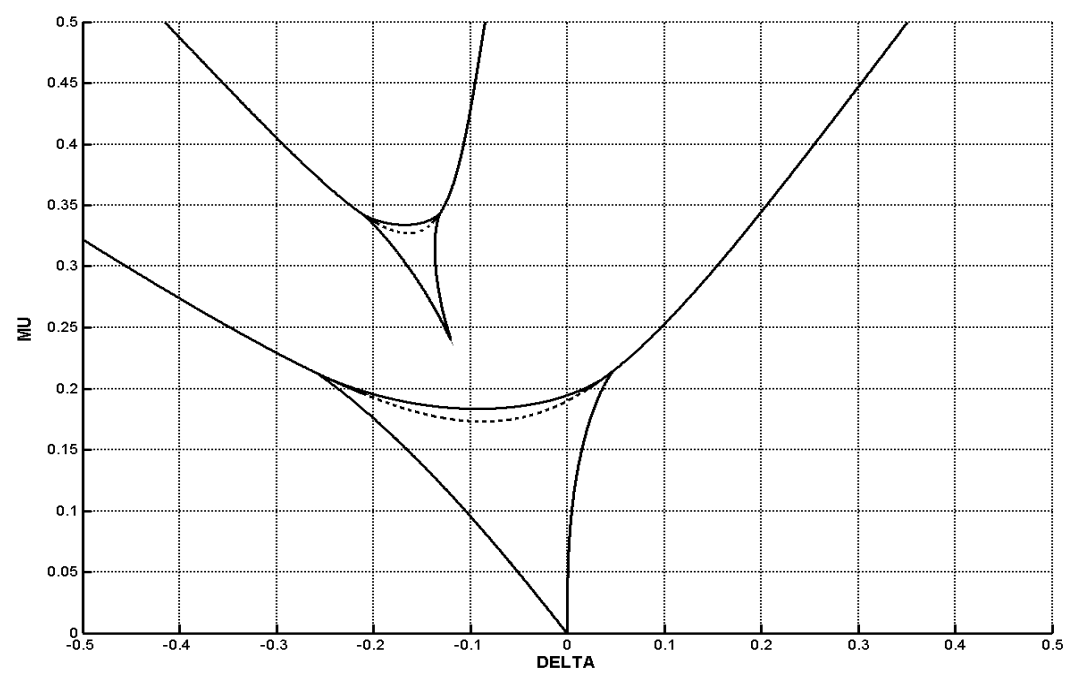

Figure 3.2: Hopf (plus saddle-node) bifurcation curves in parameter space.

To approximate the asymptotes (for large $\Delta$ and $\mu$ ) to the Hopf bifurcation curves, we go back to equation (3.19) and neglect terms that are not of the $16^{\text {th }}$ power. This leaves us with 17 of the original 81 terms in the equation. We solve the resulting equations to find $\mu$ as a function of $\Delta$. The resulting asymptote equations are

$$
\begin{gathered}
\mu=-\frac{2\left(7^{3 / 4}+\sqrt{59 \sqrt{7}-119}-4\left(7^{1 / 4}\right)\right)}{9\left(7^{1 / 4}\right)} \Delta=-0.53119 \Delta \\
\mu=\frac{2\left(-7^{3 / 4}+\sqrt{59 \sqrt{7}-119}+4\left(7^{1 / 4}\right)\right)}{9\left(7^{1 / 4}\right)} \Delta=1.13308 \Delta \\
\mu=-\frac{2\left(-7^{3 / 4}+\sqrt{59 \sqrt{7}+119}-4\left(7^{1 / 4}\right)\right)}{9\left(7^{1 / 4}\right)} \Delta=-0.78915 \Delta \\
\mu=\frac{2\left(7^{3 / 4}+\sqrt{59 \sqrt{7}+119}+4\left(7^{1 / 4}\right)\right)}{9\left(7^{1 / 4}\right)} \Delta=3.74282 \Delta
\end{gathered}
$$

These asymptotes may be useful when the issue of large coupling $(\mu \gg 1)$ in $(1.1)$ is analyzed in the future. 


\subsection{Other Bifurcation Curves}

Besides the saddle-node and Hopf bifurcation curves found in the previous two sections, there exist 6 other bifurcation curves in the parameter space that have been found my numerical investigation of the slow-flow. Four of them are approximated by the dotted lines in Figure 3.3 and their significance is discussed in the next chapter.

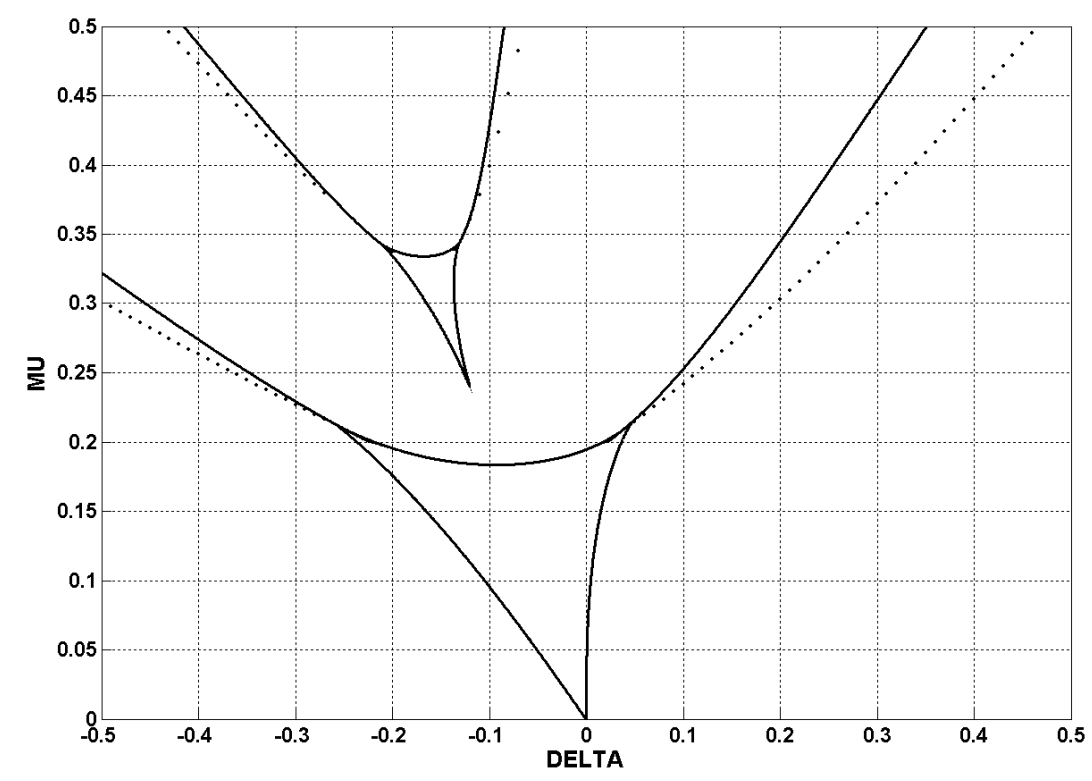

Figure 3.3: Numerically computed bifurcation curves (dotted lines) in parameter space.

Finally, there exists two last bifurcation curves in the parameter space for which we do not provide a good approximation for but whose existence is necessary for a correct description of the qualitative nature of the in-phase dynamics in the next chapter. They exist on each side of the upper triangular region, in between the top Hopf bifurcation curve and the top numerically computed bifurcation curves in Figure 3.3. A cartoon of one of these bifurcation curves is given in Figure 3.4. 


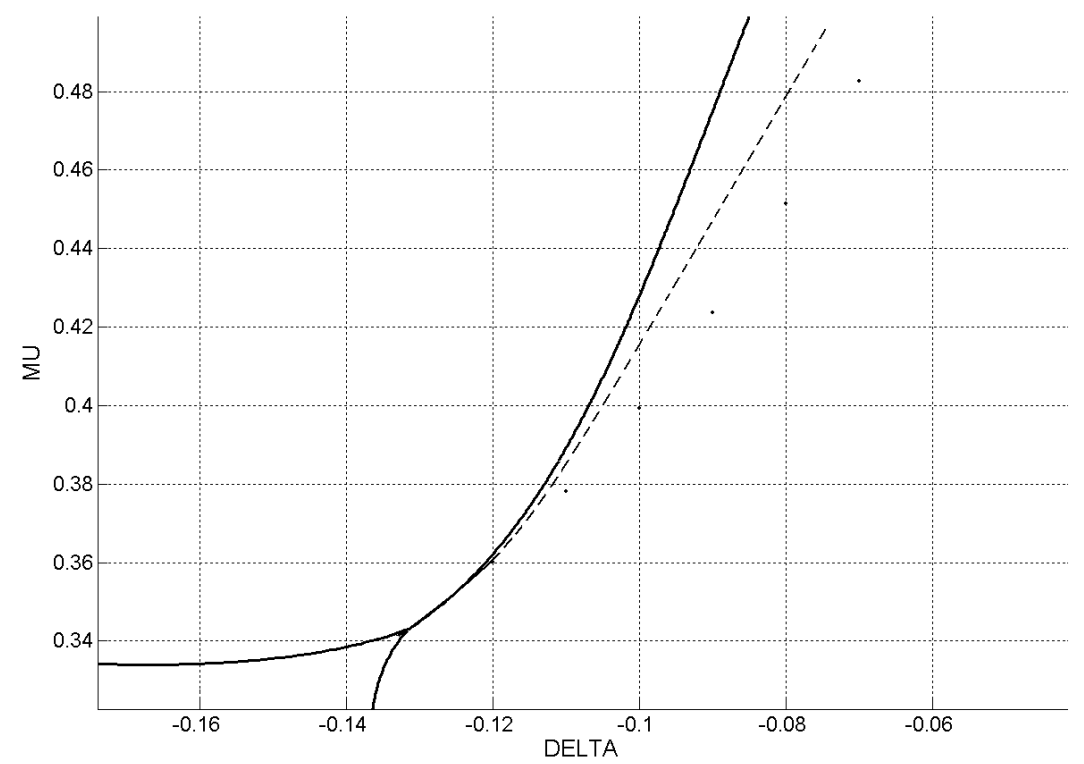

Figure 3.4: Final numerical bifurcation curves (dashed lines) in parameter space. 


\section{CHAPTER 4 THE DYNAMICS OF THE IN-PHASE MODE}

In the previous chapter we found bifurcation curves (both analytical and numerical) separating regions of distinctly different behavior in the $\Delta-\mu$ parameter space. Numerical integration of the in-phase slow-flow equations for the respective regions reveals three different types (see Figure 4.1) of dynamical behavior: (1) phase drift, (2) weakly phase-locked motion, and (3) phase-locked motion. To differentiate between these types of behavior, we will use the following definitions:

1. Phase drift occurs when the phase difference $\phi(\eta)$ between the pineal oscillator $(w)$ and the eye $(x=y)$ oscillators increases (or decreases) without bound. In the slow-flow phase space, phase drift appears as a closed curve (a limit-cycle) which is cyclic in $\phi$. Positive (negative) drift refers to the direction of the flow in $\phi$. Such motion is referred to as an LCD (limit cycle with drift).

2. A weakly phase-locked motion occurs when $\phi(\eta)$ is periodic. It is represented in the phase space by a limit cycle which is topologically distinct from an LCD. In this system weakly phase-locked motions are born in supercritical Hopf bifurcations or in folds of limit cycles. Such motion is referred to as an LCW (limit cycle with weak phase-locking).

3. Phase-locked motions are motions where $\phi(\eta)$ remains constant. These motions correspond to equilibria in the slow-flow phase space.

Figure 4.2 shows the bifurcation curves separating the parameter space into 10 labeled regions. The essential dynamical features and behavior found in each region 


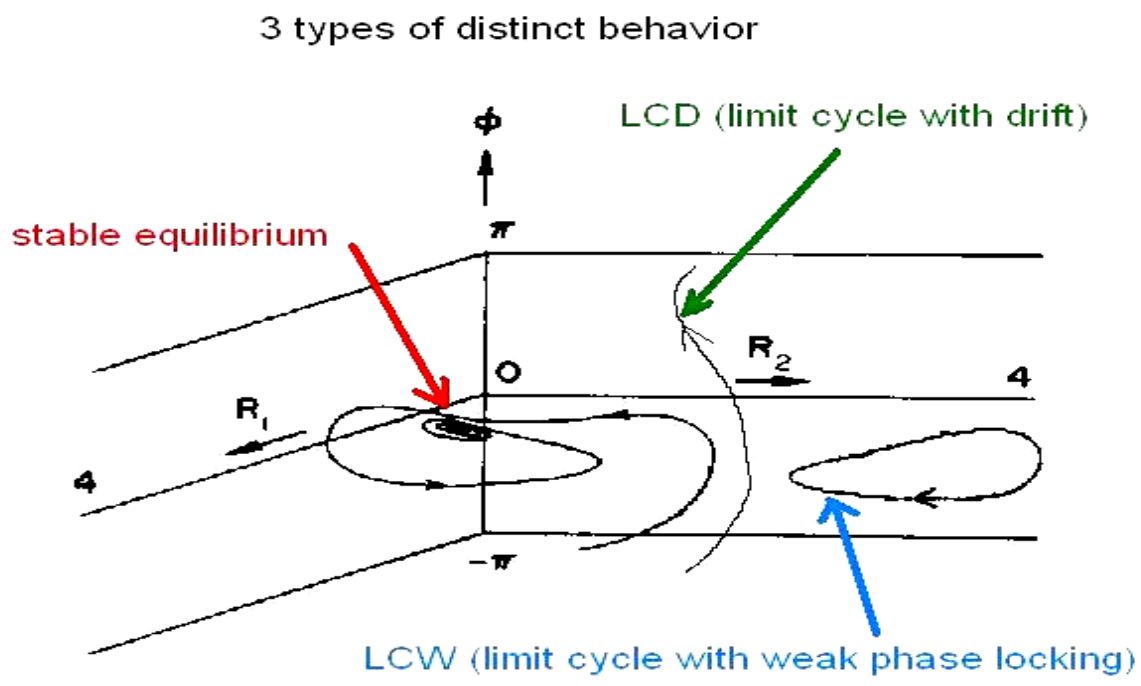

Figure 4.1: Three distinct types of dynamical behavior for the slow-flow.

are summarized as follows:

- REGION I: There exist two unstable equilibrium points and one stable LCD. All trajectories in the phase space experience negative drift.

- REGION II: There exist two unstable equilibrium points and one stable LCD. All trajectories in the phase space experience positive drift.

- REGION III: There exist one stable and three unstable equilibrium points. All trajectories tend to the stable equilibrium point.

- REGION IV: There exist one stable and one unstable equilibrium point. All trajectories tend to the stable equilibrium point.

- REGION V: There exist two stable and two unstable equilibrium points. All 
trajectories tend to one of the two stable equilibrium points.

- REGION VI: There exist two stable equilibrium points. All trajectories tend to one of the two stable equilibrium points. The also exists an unstable LCD.

- REGIONS A and D: There exist two unstable equilibrium points and a stable LCW. All trajectories approach the stable limit cycle.

- REGIONS B and C: There exist one stable and one unstable equilibrium point and a stable LCW. All trajectories are attracted to either the stable equilibrium point or to the stable LCW. In addition there exists an unstable motion which is an LCD at the region VI side of regions $\mathrm{B}$ and $\mathrm{C}$, but which is an LCW at the region IV side. The change from LCD to LCW occurs along a curve (see Figure 3.4) in each of regions B and $\mathrm{C}$ via a saddle connection bifurcation.

\subsection{Transitions Between Regions of Parameter Space}

To properly describe the dynamics of the slow-flow we explore how its dynamical features change as we cross each bifurcation curve in parameter space. We only look at bifurcation curve crossings on the right-hand side of the line $\mu=-2 \Delta$ in parameter space as the types of bifurcations represented by the bifurcation curves are the same on both sides of this line. The numerical values given for the bifurcation points are approximate and only intended to give an idea of where the numerical bifurcation curves are. 


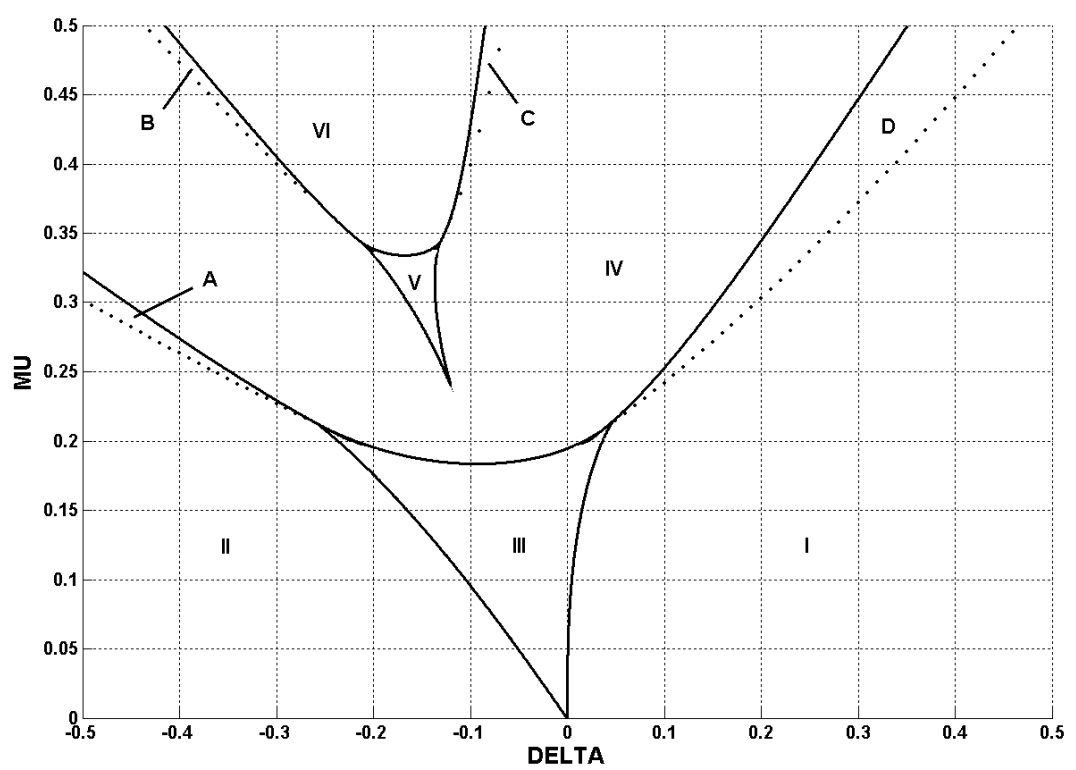

Figure 4.2: Regions of parameter space exhibiting distinctly different dynamical behavior.

\section{Region I to Region III to Region IV}

Starting in Region I, we fix $\Delta=0.03$ and travel in the positive $\mu$-direction (see Figure 4.3). At point A the phase space is dominated by a stable LCD and two unstable equilibrium points, as shown in part A of Figure 4.4. Continuing upwards to point B, we cross the saddle-node bifurcation curve (3.11) into Region III. It is along this bifurcation curve that two new equilibria, one stable and the other unstable, are born in an infinite-period bifurcation taking place along the stable LCD (part B of Figure 4.4). As $\mu$ is increased even more the distance between the two new equilibria increases and two of the unstable equilibria approach one another. Finally, at point D in Figure 4.3 we cross the saddle-node bifurcation curve (3.11) again, this time moving into Region IV. Along this curve one of the two original unstable equilibrium points collides with the unstable equilibrium 
point born in the infinite-period bifurcation in a saddle-node bifurcation, leaving only one stable and one unstable equilibria as we enter Region IV (part E of Figure $4.4)$.

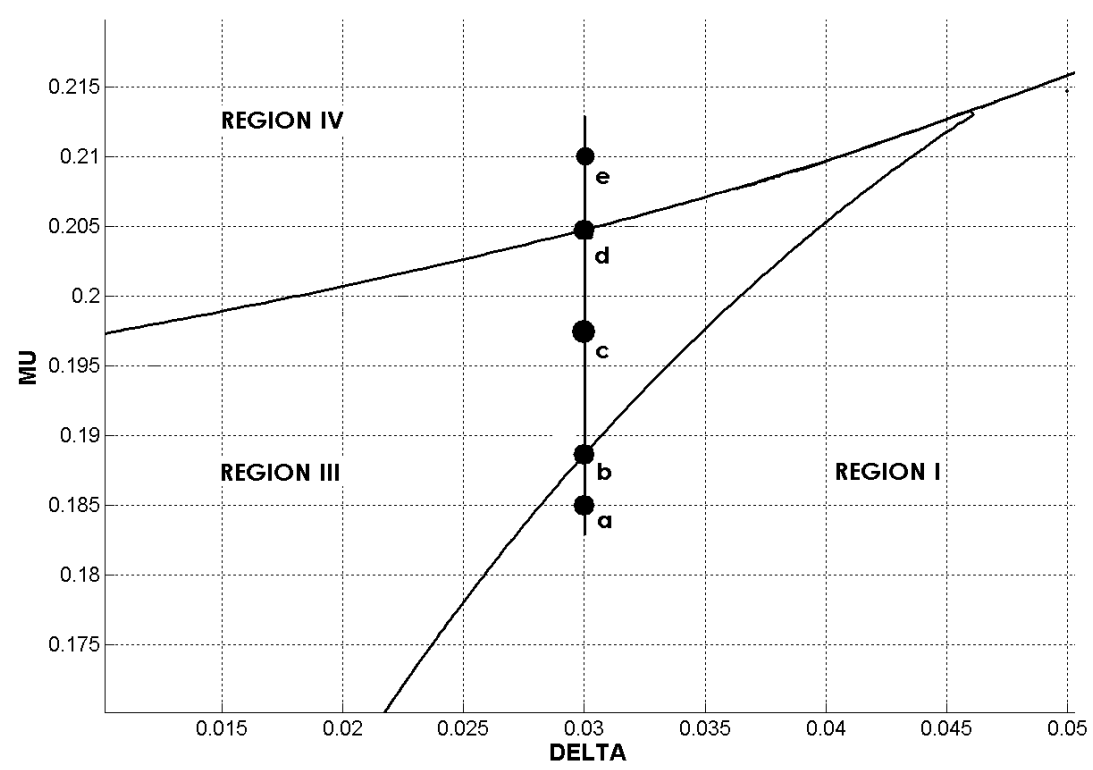

Figure 4.3: Path followed when crossing from Region I into Region III and then into Region IV. 


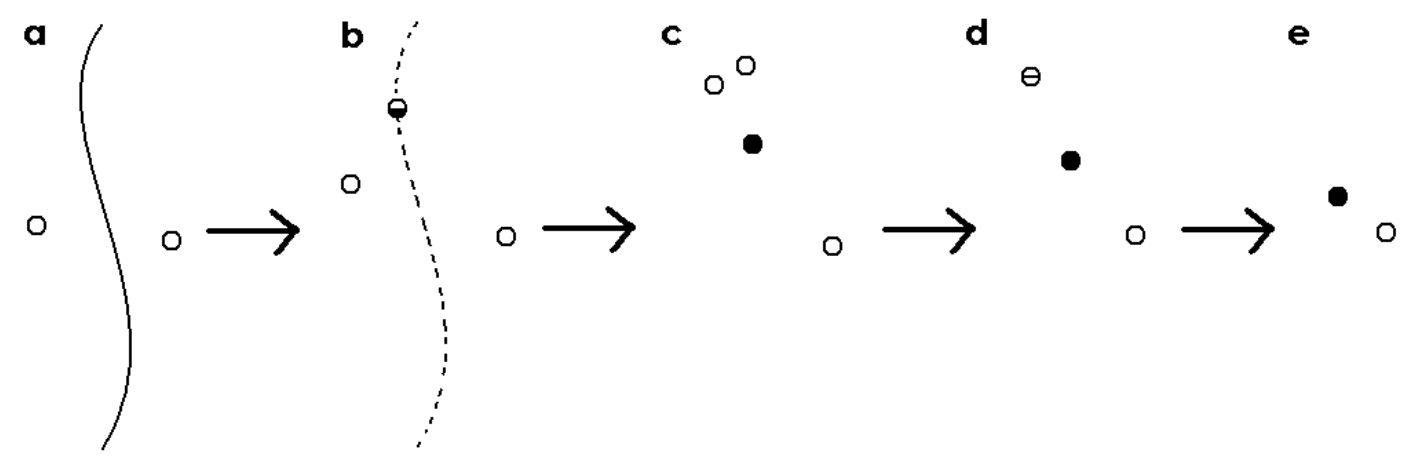

Figure 4.4: 2-dimensional cartoon of 3-dimensional bifurcations: (a) Two unstable equilibrium points separated by a stable LCD. (b) An infinite-period bifurcation occurs along stable LCD creating new stable and unstable equilibrium points. Dashed line represents structurally unstable (bifurcating) LCD. (c) Two unstable equilibrium points converge on each other. (d) A saddle-node bifurcation occurs between two unstable equilibrium points. (e) One stable and one unstable equilibrium point remain. 


\section{Region I to Region D to Region IV}

Starting again in Region I (point A in Figure 4.5) we have a stable LCD and two unstable equilibrium points. As we move in the positive $\mu$-direction towards Region IV, we hit a new bifurcation curve at approximately $(\Delta, \mu)=(0.2,0.303)$. This new curve, for which we have no analytical formula, represents saddle-node connections (heteroclinic bifurcations) where the stable LCD interacts with the non-isolated equilibria found on the singular surfaces $R_{1}=0$ and $R_{3}=0$ (see Chapter 5 or [4] for a more thorough description of this bifurcation). As we pass into region D, the saddle-connection breaks and we are left with a stable LCW with two unstable equilibrium points (part C of Figure 4.6). Moving through region D to point D we pass through the Hopf bifurcation curve (3.19) where one of the unstable equilibria undergoes a supercritical Hopf bifurcation, destroying the LCW. Thus we are left with one stable and one unstable equilibria in Region IV. 


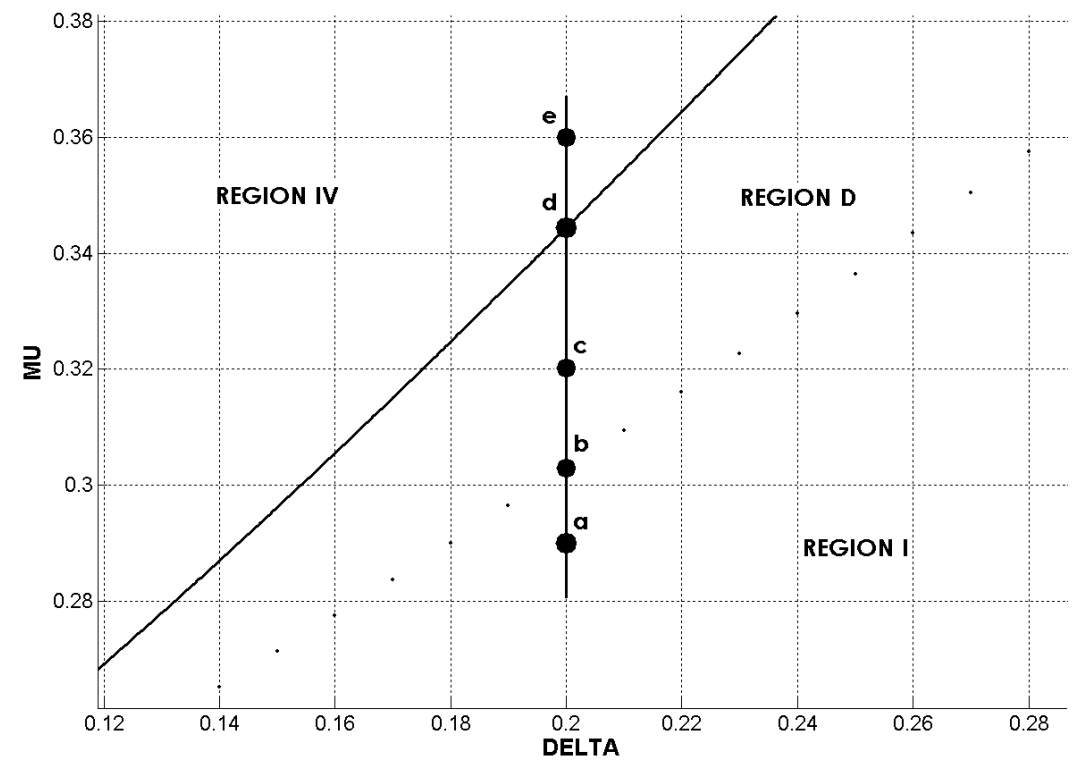

Figure 4.5: Path followed when crossing from Region I into Region D and then into Region IV. 


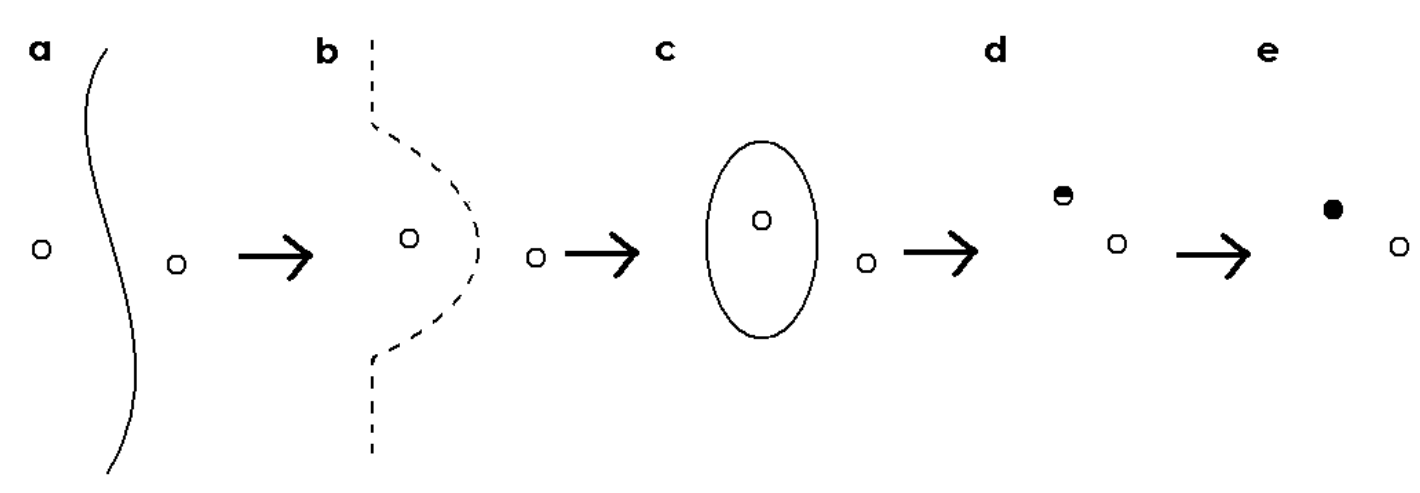

Figure 4.6: 2-dimensional cartoon of 3-dimensional bifurcations: (a) Two unstable equilibrium points separated by a stable LCD. (b) A heteroclinic (saddle-connection) bifurcation occurs. Dashed line represents structurally unstable (bifurcating) LCD. (c) After the saddle-connection bifurcation the stable LCD becomes a stable LCW surrounding an unstable equilibrium. (d) The stable LCW decreases in size until a super-critical Hopf bifurcation happens. (e) One stable and one unstable equilibrium point remain. 


\section{Region VI to Region V to Region IV}

Beginning this time in Region VI we have two stable equilibria and one unstable LCD (part A of Figure 4.8). Moving diagonally to point B in Figure 4.7, we cross the upper part of the upper-triangle portion of the saddle-bifurcation curve (3.11), where two unstable equilibria appear in an infinite-period bifurcation along the unstable LCD. Continuing through Region V the equilibrium points separate until at point $\mathrm{D}$ in Figure 4.7 a stable and unstable equilibria collide in a saddle-node bifurcation, leaving one unstable and one stable equilibria in Region IV.

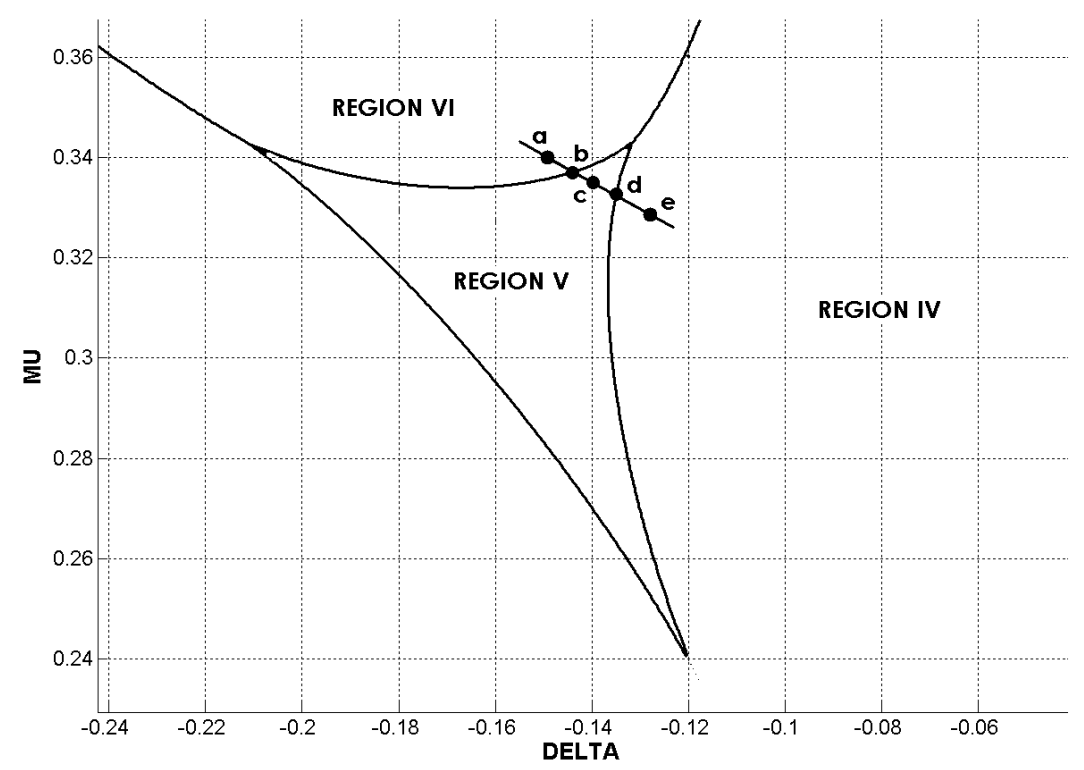

Figure 4.7: Path followed when crossing from Region IV into Region V and then into Region VI. 


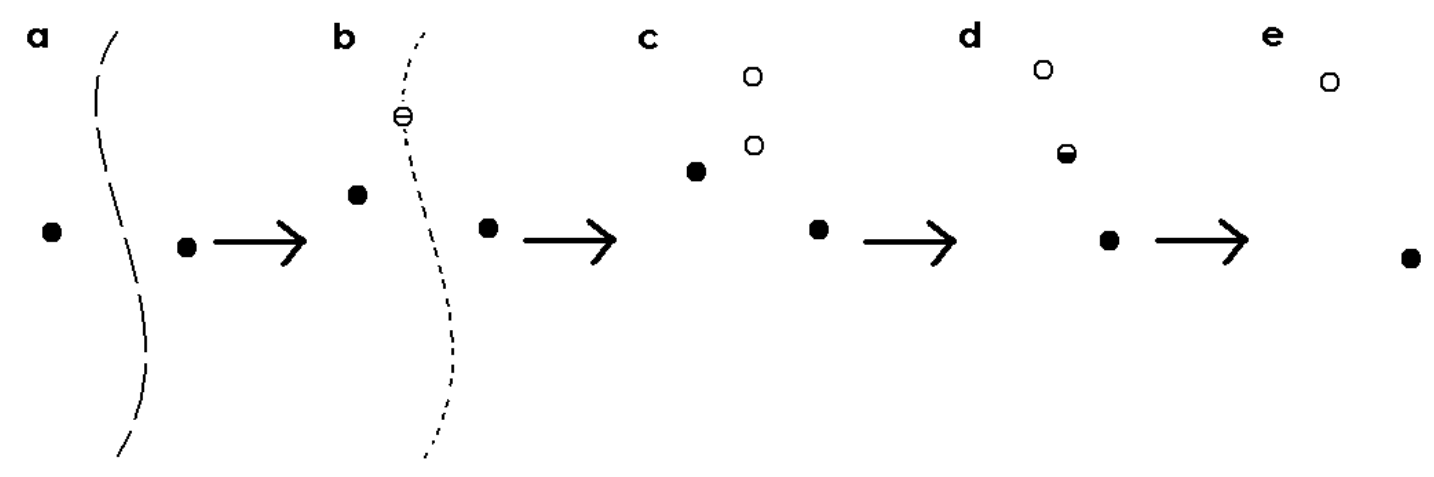

Figure 4.8: 2-dimensional cartoon of 3-dimensional bifurcations: (a) Two stable equilibrium points separated by an unstable LCD. (b) An infinite-period bifurcation occurs along the unstable LCD creating two new unstable equilibrium points. Dashed line represents structurally unstable (bifurcating) LCD. (c) A stable and unstable equilibrium point converge on each other. (d) A saddlenode bifurcation occurs between a stable and unstable equilibrium point. (e) One stable and one unstable equilibrium point remain. 


\section{Region IV to Region C to Region VI}

Beginning in Region IV, we start at $(\Delta, \mu)=(-0.06, .48)$ and this time decrease $\Delta$. As we approach Region $\mathrm{C}$ at point $\mathrm{B}$ in Figure $4.9(\Delta=-.0708)$, a global bifurcation occurs - a fold of LCWs, giving us one stable and one unstable equilibria plus one stable and one unstable LCW as we enter region C. Continuing through Region C, the stable LCW decreases in size around the unstable equilibria, while the unstable LCW increases in size. Midway through, at pont D in Figure 4.9, the unstable LCW forms a heteroclinic trajectory with the singular surface at approximately $\Delta=-.085$, after which the unstable LCW becomes an unstable LCD (see parts D and E of Figure 4.9). When we cross the Hopf curve (3.19) going into Region VI, the stable LCW shrinks down to the unstable equilibria and a supercritical Hopf bifurcation takes place. Thus we are left with two stable equilibria and an unstable LCD in Region VI. 


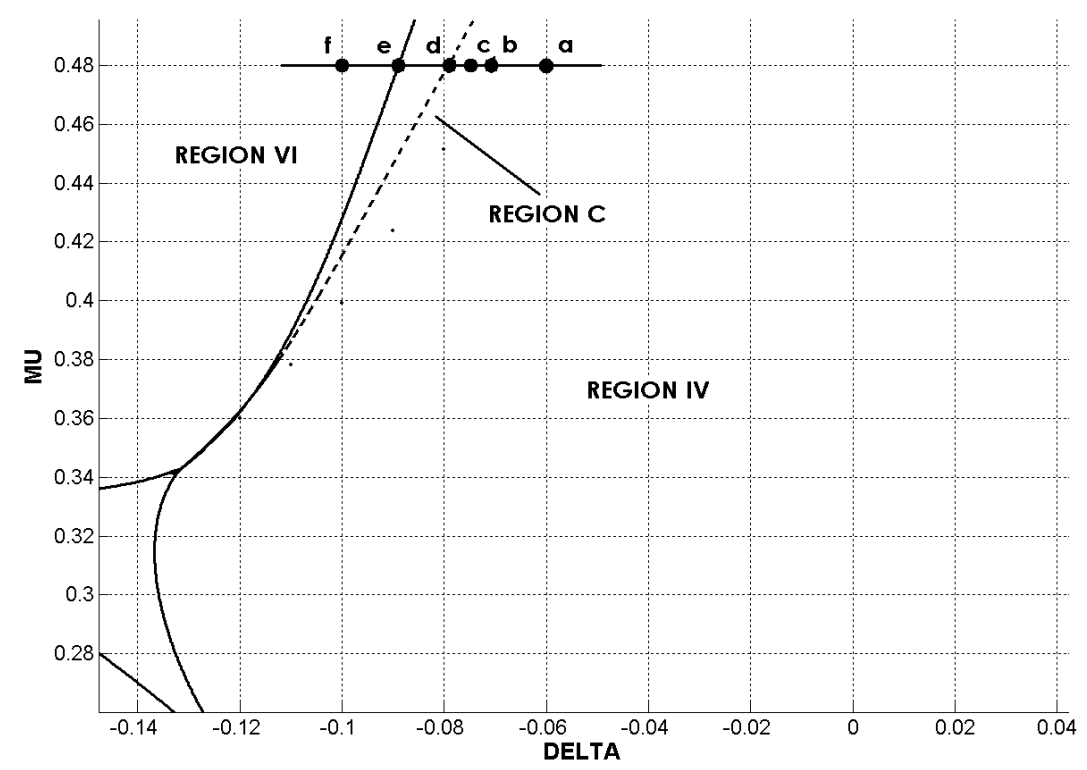

Figure 4.9: Path followed when crossing from Region IV into Region $\mathrm{C}$ and then into Region VI. 


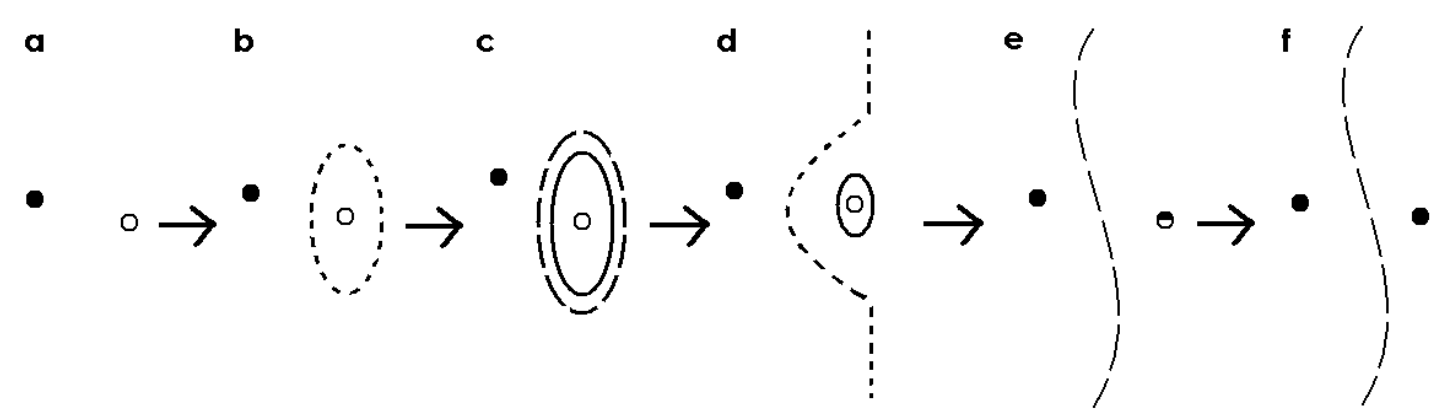

Figure 4.10: 2-dimensional cartoon of 3-dimensional bifurcations: (a) One stable and one unstable equilibrium point exist. (b) A fold of LCWs occurs around the unstable equilibrium point. Dashed line represents structurally unstable (bifurcating) LCW. (c) As the LCWs separate, the stable LCW shrinks in size while the unstable LCW grows. (d) A heteroclinic (saddle-connection) bifurcation occurs. Dashed line represents structurally unstable (bifurcating) LCW. (e) A Hopf bifurcation occurs as the stable LCW collapses to the unstable equilibrium point. (f) Two stable equilibria exist and are separated by an unstable LCD. 


\subsection{Analysis of the Slow-Flow Dynamics}

In terms of the biological applications of this work, we are interested in regions in parameter space where there exist a stable equilibrium point of the slow-flow. Mathematically this corresponds to a phase-locked in-phase motion of the original six-dimensional problem (1.1). Biologically, it corresponds to the in-phase synchronized behavior of the circadian rhythms in each of the eyes (the $x$ and $y$ oscillators) in relation to that of the extra-ocular circadian system (the $w$ oscillator). As stated in the introduction, the existence of a phase-locked in-phase motion is important because it has been conjectured that if the melatonin rhythms in the retinae of Japanese quails were to be the primary circadian pacemaker they would need to be (i) tightly coupled and (ii) in-phase so to produce a coherent rhythm. [10]

In Figure 4.10 the regions of parameter space where such synchronized motions exist have been shaded. We see that for a given coupling strength $\mu$, synchronization can only occur if the natural frequency of the extra-ocular oscillator, $1+\epsilon \Delta$, is within a certain detuning range, $\Delta_{\min } \leq \Delta \leq \Delta_{\max }$. This is commonly referred to as the range of synchronization or entrainment. Inversely, we can state that given an extra-ocular oscillator with a natural frequency slightly detuned from that of the eye oscillators, there exists a minimum coupling strength $\mu_{\text {min }}$ necessary in order to synchronize the rhythms. These results agree with those from earlier studies on the synchronization of coupled (or forced) nonlinear oscillators (see [11] for an example).

We also note that in Regions V, VI, B, and C of Figure 4.2 there exist multiple stable in-phase phase-locked motions. While the biological significance of regions 


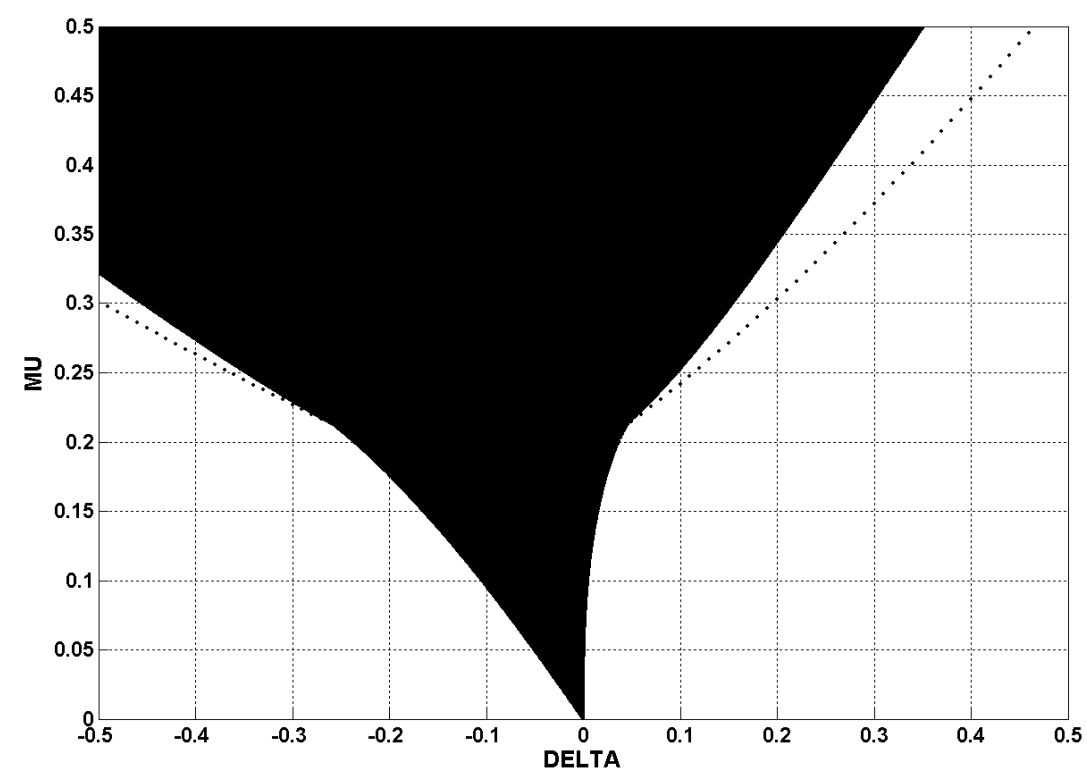

Figure 4.11: Regions of parameter space where stable phase-locked motion is possible.

of multiple stable behavior existing primarily when the eye oscillators have a higher frequency (and thus a shorter period) than that of the extra-ocular circadian system are unknown, it would indicate a possible dependence for steady-state motion on the initial conditions for the circadian oscillators and possible hysteresis. 
CHAPTER 5

THE SLOW-FLOW ON SINGULAR SURFACES

In the 3-dimensional in-phase slow-flow phase space the surfaces $R_{1}=0$ and $R_{3}=0$ are singular surfaces in the sense that as a trajectory approaches either of these surfaces the slow-flow equations governing its evolution become ill-defined (both $R_{1}$ and $R_{3}$ are found in the denominator of terms in equations (3.3)). Therefore the analysis of the dynamics on these surfaces requires a more sophisticated mathematical analysis.

\subsection{Slow-Flow on the Singular Surface $R_{1}=0$}

The process of finding the slow-flow along a singular surface is called scaling the singularity. To find the slow-flow on the singular plane $R_{1}=0$ we change independent variables to (following [4])

$$
d \tau=\frac{d \eta}{R_{1}}
$$

In terms of our original independent time variable $t$

$$
d \tau=\frac{\epsilon}{R_{1}} d t
$$

Substituting (5.1) into the slow-flow equations (3.3) we get the following scaled system

$$
\begin{gathered}
\frac{d R_{1}}{d \tau}=-\frac{\mu R_{1} R_{3}}{2} \sin \phi+\frac{R_{1}^{2}}{2}\left(1-\frac{R_{1}^{2}}{4}\right) \\
\frac{d R_{3}}{d \tau}=\mu R_{1}^{2} \sin \phi+\frac{R_{1} R_{3}}{2}\left(1-\frac{R_{3}^{2}}{4}\right) \\
\frac{d \phi}{d \tau}=\frac{\mu}{2}\left[\left(\frac{2 R_{1}^{2}}{R_{3}}-R_{3}\right) \cos \phi-R_{1}\right]-R_{1} \delta
\end{gathered}
$$


Substituting $R_{1}=0$ into (5.3) gives the dynamics on the singular surface

$$
\begin{gathered}
\frac{d R_{1}}{d \tau}=0 \\
\frac{d R_{3}}{d \tau}=0 \\
\frac{d \phi}{d \tau}=-\frac{\mu R_{3}}{2} \cos (\phi)
\end{gathered}
$$

Thus $R_{1}$ and $R_{3}$ remain constant on the singular surface $R_{1}=0$ while only the phase-difference $\phi$ changes. Furthermore, there exist two 1-dimensional subspaces (lines) of non-isolated equilibria at $\phi= \pm \frac{\pi}{2}$ (assuming $\mu>0$ and ignoring the even more singular case when $R_{3}=0$ ). To find the stability of the non-isolated equilibria within the singular plane, with $\bar{R}_{3}$ denoting the $R_{3}$ value of an equilibrium point, we solve the first-order ODE $(5.4 \mathrm{c})$ to get

$$
\ln \left(\frac{1-\sin (\phi)}{1+\sin (\phi)}\right)=\mu \bar{R}_{3} \tau+c_{1}
$$

where $c_{1}$ is a constant. Solving for $\sin (\phi)$ gives

$$
\sin (\phi)=\frac{1-c_{2} e^{\mu \bar{R}_{3} \tau}}{1+c_{2} e^{\mu \bar{R}_{3} \tau}}
$$

where $c_{2}$ is a new constant dependent on $c_{1}$. As $\tau \rightarrow \infty, \sin (\phi) \rightarrow-1$ by the right-hand side of (5.6) and thus $\phi \rightarrow-\frac{\pi}{2}$. Likewise, as $\tau \rightarrow-\infty, \sin (\phi) \rightarrow 1$ and thus $\phi \rightarrow \frac{\pi}{2}$. Therefore, on the singular surface $R_{1}=0$ the subspace corresponding to $\phi=-\frac{\pi}{2}$ is stable and $\phi=\frac{\pi}{2}$ is unstable (note that this may not hold when we consider stability in the larger 3-dimensional phase-space). This result can be shown graphically as a flow on the $\phi$-circle (see Figure 5.1). To further understand the nature of the dynamics near these nonisolated equilibrium points we linearize around the equilibria and solve for the linearized slow-flow. 


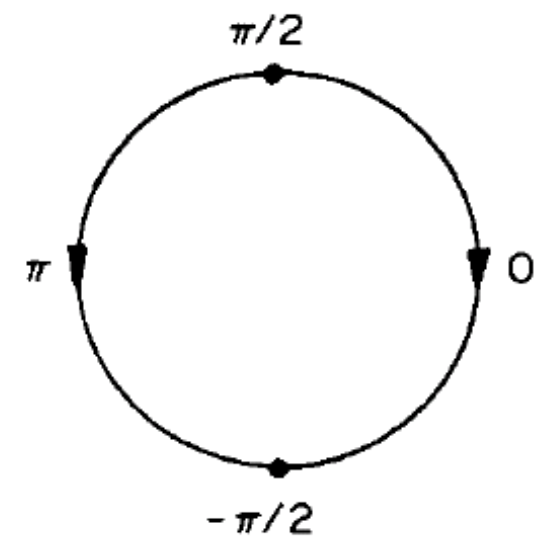

Figure 5.1: Flow on the $\phi$-circle for $R_{1}=0$ [5].

The Jacobian of the scaled system (5.3) is

$$
\left[\begin{array}{ccc}
-\frac{\mu \sin (\phi) R_{3}+R_{1}^{3}-2 R_{1}}{2} & -\frac{\mu \sin (\phi) R_{1}}{2} & -\frac{\mu \cos (\phi) R_{1} R_{3}}{2} \\
-\frac{R_{3}^{3}-4 R_{3}-16 \mu \sin (\phi) R_{1}}{8} & -\frac{R_{1}\left(3 R_{3}^{2}-4\right)}{8} & \mu \cos (\phi) R_{1}^{2} \\
-\frac{\mu R_{3}+2 \Delta R_{3}-4 \mu \cos (\phi) R_{1}}{2 R_{3}} & -\frac{\mu \cos (\phi)\left(R_{3}^{2}+2 R_{1}^{2}\right)}{2 R_{3}^{2}} & \frac{\mu \sin (\phi)\left(R_{3}^{2}-2 R_{1}^{2}\right)}{2 R_{3}}
\end{array}\right]
$$

Evaluating the Jacobian at equilibrium points on the lines $\left(R_{1}, R_{3}, \phi\right)=\left(0, \bar{R}_{3}, \pm \frac{\pi}{2}\right)$ gives

$$
\left[\begin{array}{ccc}
\mp \frac{\mu \bar{R}_{3}}{2} & 0 & 0 \\
\frac{\bar{R}_{3}}{2}\left(1-\frac{\bar{R}_{3}^{2}}{4}\right) & 0 & 0 \\
-\frac{\mu}{2}-\Delta & 0 & \pm \frac{\mu \bar{R}_{3}}{2}
\end{array}\right]
$$

We can now find the eigenvalues and eigenvectors associated with the Jacobian matrix and solve for the linearized slow-flow near the equilibria.

$$
\left[\begin{array}{l}
R_{1}(\tau) \\
R_{3}(\tau) \\
\phi(\tau)
\end{array}\right]=k_{1}\left[\begin{array}{l}
0 \\
1 \\
0
\end{array}\right]+k_{2}\left[\begin{array}{l}
0 \\
0 \\
1
\end{array}\right] e^{ \pm \frac{\mu \bar{R}_{3}}{2} \tau}+k_{3}\left[\begin{array}{c}
1 \\
\pm \frac{\bar{R}_{3}^{2}-4}{4 \mu} \\
\pm \frac{\mu+2 \Delta}{2 \mu R_{3}}
\end{array}\right] e^{\mp \frac{\mu \overline{R_{3}}}{2} \tau}
$$


The $\left[\begin{array}{lll}0 & 1 & 0\end{array}\right]^{T}$ eigenvector and its eigenvalue $\lambda=0$ are associated with the singular nature of the equilibria and their neutral stability in the 3-dimensional phasespace. The $\left[\begin{array}{lll}0 & 0 & 1\end{array}\right]^{T}$ eigenvector corresponds to the flow within the $R_{1}=0$ singular surface. The positive eigenvalue $\lambda=\frac{\mu \overline{R_{3}}}{2}$ associated with the line of nonisolated equilibria at $\phi=\frac{\pi}{2}$ once again shows that this line is unstable in the singular surface. Likewise, at $\phi=-\frac{\pi}{2}$ the line of equilibria is stable due to its negative eigenvalue. The final eigenvector $\left[\begin{array}{lll}1 & \pm \frac{{\overline{R_{3}}}^{2}-4}{4 \mu} & \pm \frac{\mu+2 \Delta}{2 \mu \bar{R}_{3}}\end{array}\right]^{T}$ describes how trajectories enter (exit) the singular surface from (to) the rest of the phase space. Because of the nonisolated nature of the equilibria the eigendirections form a 2dimensional manifold in the phase space [4]. The negative eigenvalue $\lambda=-\frac{\mu \overline{R_{3}}}{2}$ associated with the line of nonisolated equilibria at $\phi=\frac{\pi}{2}$ shows that trajectories enter the singular surface along this manifold and exit via the manifold originating from the line $\phi=-\frac{\pi}{2}$. Note that assuming $\mu>0$ and $R_{3}>0$ the stability of the nonisolated equilibria within the singular plane does not change.

\subsection{Slow-Flow on the Singular Surface $R_{3}=0$}

To find the slow-flow on the singular plane $R_{3}=0$ we once again change independent variables, this time defining the new time variable as

$$
d \tau=\frac{d \eta}{R_{3}}
$$

Substituting this into the slow-flow equations (3.3) we get the following scaled system

$$
\begin{gathered}
\frac{d R_{1}}{d \tau}=-\frac{\mu R_{3}^{2}}{2} \sin \phi+\frac{R_{1} R_{3}}{2}\left(1-\frac{R_{1}^{2}}{4}\right) \\
\frac{d R_{3}}{d \tau}=\mu R_{1} R_{3} \sin \phi+\frac{R_{3}^{2}}{2}\left(1-\frac{R_{3}^{2}}{4}\right)
\end{gathered}
$$




$$
\frac{d \phi}{d \tau}=\frac{\mu}{2}\left[\left(2 R_{1}-\frac{R_{3}^{2}}{R_{1}}\right) \cos \phi-R_{3}\right]-R_{3} \Delta
$$

Substituting $R_{3}=0$ into (5.11) gives the dynamics on this singular surface as

$$
\begin{gathered}
\frac{d R_{1}}{d \tau}=0 \\
\frac{d R_{3}}{d \tau}=0 \\
\frac{d \phi}{d \tau}=\mu R_{1} \cos \phi
\end{gathered}
$$

Thus $R_{1}$ and $R_{3}$ remain constant this singular surface as well, while only the phasedifference $\phi$ changes. Furthermore, there exist two 1-dimensional subspaces (lines) of non-isolated equilibria at $\phi= \pm \frac{\pi}{2}$ (assuming $\mu>0$ and ignoring the even more singular case when $R_{1}=0$ ). To find the stability of the non-isolated equilibria within the singular plane we solve the first-order ODE (5.12c) to get

$$
\ln \left(\frac{1+\sin (\phi)}{1-\sin (\phi)}\right)=2 \mu \bar{R}_{1} \tau+c_{1}
$$

where $c_{1}$ is a constant and $\bar{R}_{1}$ a given equilibrium value. Solving for $\sin (\phi)$ gives

$$
\sin (\phi)=\frac{c_{2} e^{2 \mu \bar{R}_{1} \tau}-1}{c_{2} e^{2 \mu \bar{R}_{1} \tau}+1}
$$

where $c_{2}$ is a new constant dependent on $c_{1}$. Performing the same analysis as in the previous section, as $\tau \rightarrow \infty, \sin (\phi) \rightarrow 1$ by the right-hand side of (5.14) and thus $\phi \rightarrow \frac{\pi}{2}$. Likewise, as $\tau \rightarrow-\infty, \sin (\phi) \rightarrow-1$ and thus $\phi \rightarrow-\frac{\pi}{2}$. Therefore, on the singular surface $R_{3}=0$ the subspace corresponding to $\phi=\frac{\pi}{2}$ is stable and $\phi=-\frac{\pi}{2}$ is unstable (note that this may not hold when we consider stability in the larger 3-dimensional phase-space). Thus these 1-dimensional subspaces of nonisolated equilibria corresponding to $\phi= \pm \frac{\pi}{2}$ have the opposite stability within this singular plane as they do within the other singular plane $R_{1}=0$ (see Figure $5.2)$. 


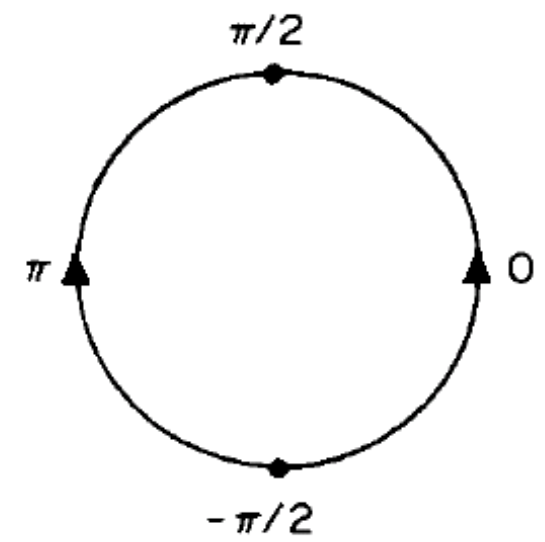

Figure 5.2: Flow on the $\phi$-circle for $R_{3}=0$.

To further understand the nature of these nonisolated equilibrium points we linearize around the equilibria and solve for the linearized slow-flow. The Jacobian of the scaled system $(5.11)$ is

$$
\left[\begin{array}{ccc}
-\frac{3 R_{1}^{2}-4}{8} & -\frac{8 \mu \sin \phi R_{3}+R_{1}^{3}-4 R_{1}}{8} & -\frac{\mu \cos \phi R_{3}^{2}}{2} \\
\mu \sin \phi R_{3} & -\frac{R_{3}^{3}-2 R_{3}-2 \mu \sin \phi R_{1}}{2} & \mu \cos \phi R_{1} R_{3} \\
\frac{\mu \cos \phi R_{3}^{2}+2 \mu \cos \phi R_{1}^{2}}{2 R_{1}^{2}} & -\frac{2 \mu \cos \phi R_{3}+(\mu+2 \Delta) R_{1}}{2 R_{1}} & \frac{\mu \sin \phi R_{3}^{2}-2 \mu \sin \phi R_{1}^{2}}{2 R_{1}}
\end{array}\right]
$$

Evaluating the Jacobian at equilibrium points on the lines $\left(R_{1}, R_{3}, \phi\right)=\left(\bar{R}_{1}, 0, \pm \frac{\pi}{2}\right)$ gives

$$
\left[\begin{array}{ccc}
0 & \frac{R_{1}}{2}\left(1-\frac{R_{1}^{2}}{4}\right) & 0 \\
0 & \pm \mu R_{1} & 0 \\
0 & -\frac{\mu}{2}-\delta & \mp \mu R_{1}
\end{array}\right]
$$

We can now find the eigenvalues and eigenvectors associated with the Jacobian 
(5.15) and solve for the linearized slow-flow near the equilibria, giving

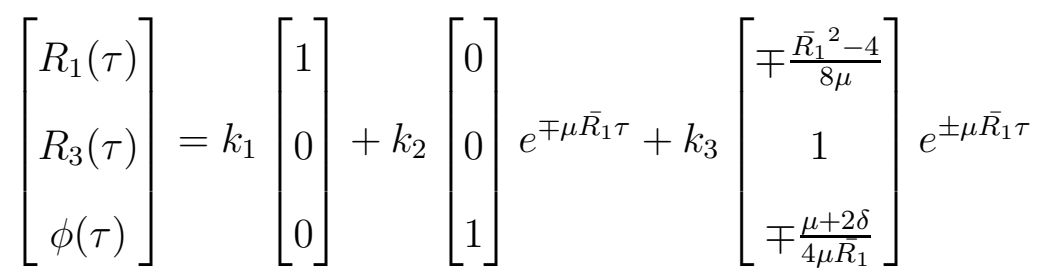

The $\left[\begin{array}{lll}1 & 0 & 0\end{array}\right]^{T}$ eigenvector and its eigenvalue $\lambda=0$ are associated with the singular nature of the equilibria and their neutral stability within the 3-dimensional phase-space. The $\left[\begin{array}{lll}0 & 0 & 1\end{array}\right]^{T}$ eigenvector corresponds to the flow within the $R_{3}=0$ singular surface. The negative eigenvalue $\lambda=-\mu \bar{R}_{1}$ associated with the line of nonisolated equilibria at $\phi=\frac{\pi}{2}$ shows once again that this line is stable in the singular surface. Likewise, at $\phi=-\frac{\pi}{2}$ the line of equilibria is unstable due to its positive eigenvalue. The final eigenvector $\left[\begin{array}{lll}\mp \frac{\bar{R}_{1}{ }^{2}-4}{8 \mu} & 1 & \mp \frac{\mu+2 \delta}{4 \mu \bar{R}_{1}}\end{array}\right]^{T}$ again describes how trajectories enter (exit) the singular surface from (to) the rest of the phase space and all the eigendirections taken together form a 2-dimensional manifold in the phase-space. The positive eigenvalue $\lambda=\mu \bar{R}_{1}$ associated with the line of nonisolated equilibria at $\phi=\frac{\pi}{2}$ shows that trajectories leave the singular surface along this manifold and enter via the manifold originating from the line $\phi=-\frac{\pi}{2}$. Figure 5.3 shows the nature of the slow-flow on the singular planes for two of the nonisolated equilibria.

\subsection{Further Analysis of the Singular Dynamics}

Now that we have found the flows within the singular surfaces and the means by which a trajectory can enter (exit) the singular surfaces from (to) the rest of the phase space, we discuss two fundamental ways in which these dynamics affect the global dynamics found in the phase space. 


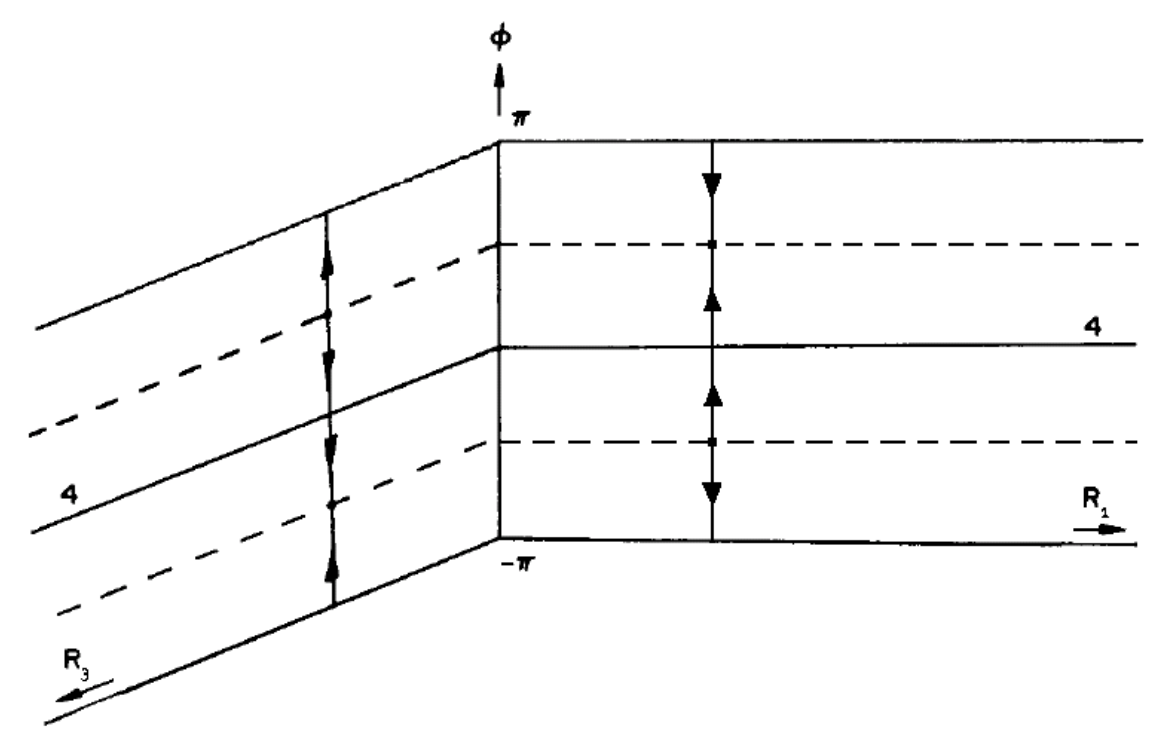

Figure 5.3: Examples of flows on the two singular planes $R_{1}=0$ and $R_{3}=0$.

First, the two 2-dimensional manifolds formed by the eigenvectors of the nonisolated equilibria on a singular surface play a role in the bifurcation between LCDs and LCWs (along the numerically computed bifurcation curves separating Regions I and D and Regions II and A shown in Figure 4.2). The mechanism by which the transition is accomplished was previously reported on in the problem of two weakly-coupled van der Pol oscillators [4]. Figure 5.4 shows a trajectory experiencing negative phase-drift when $(\Delta, \mu)=(0.2,0.3024)$ (just on the Region I side of the previously mentioned numerical curve).

One can see that the phase-drift trajectory lies on the outside of the pair of 2dimensional manifolds emanating from the singular surface $R_{3}=0$. If we now increase $\mu$ to a value of 0.304 we see (Figure 5.5) that the there now exists a stable LCW limit-cycle that lies entirely within the pair of 2-dimensional manifolds. 


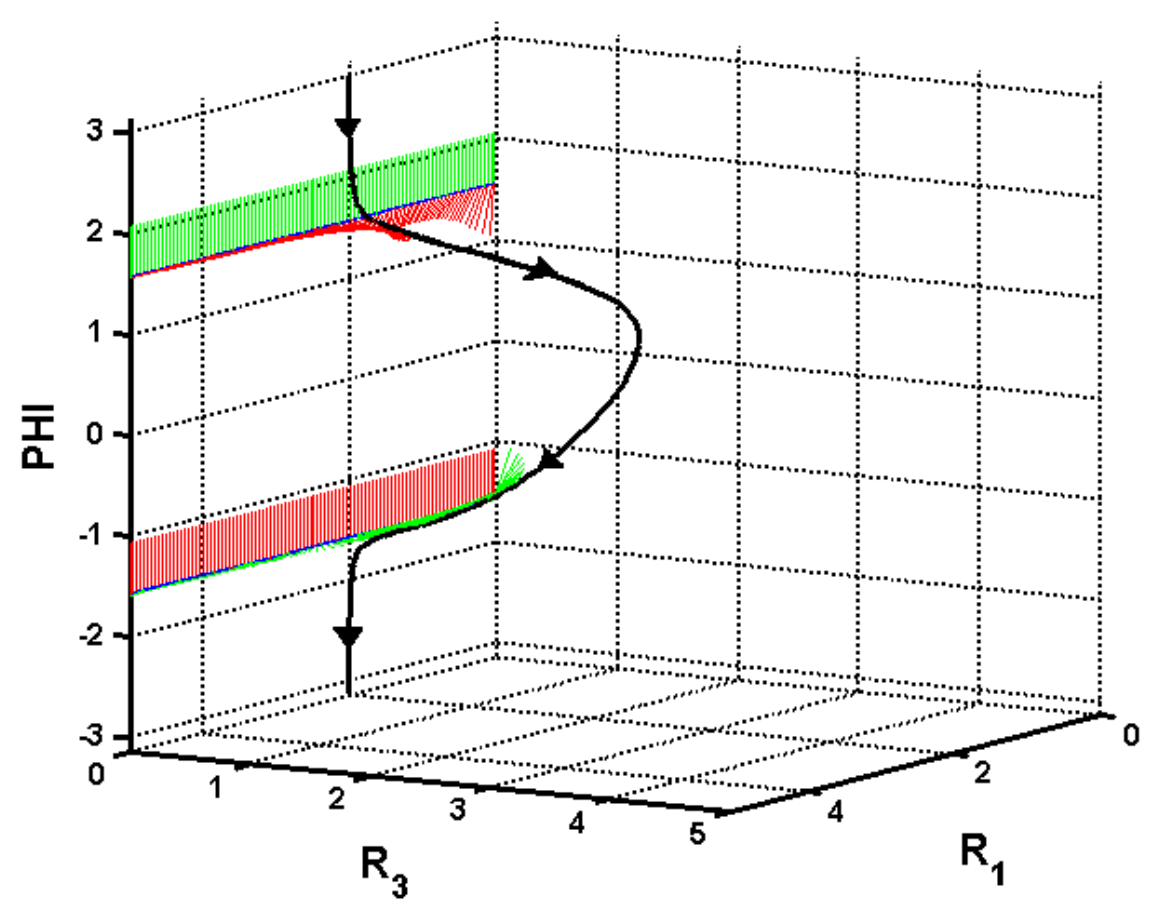

Figure 5.4: Example of stable negative drift when $(\Delta, \mu)=(0.2,0.3024)$

Thus as a stable LCD bifurcates to an LCW there exists, by continuity, a saddle connection [4].

Secondly, knowing how trajectories enter and leave the singular surfaces helps us piece together a little of what occurs along the singular line $\mu=-2 \Delta$ in parameter space. As mentioned previously, this line is not technically a bifurcation curve in the 3-dimensional in-phase problem (3.3), but it does appear to represent a bifurcation curve in the larger 5-dimensional slow-flow (2.10).

Looking back at the linear singular slow-flow solutions (5.9) and (5.16) near the nonisolated equilibria, and in particular the third eigenvectors which permit entry and exit from the singular surfaces, we note that along the line $\mu=-2 \Delta$ they become $\left[\begin{array}{lll}1 & \pm \frac{\bar{R}_{3}^{2}-4}{4 \mu} & 0\end{array}\right]^{T}$ and $\left[\begin{array}{lll}\mp \frac{\bar{R}_{1}^{2}-4}{8 \mu} & 1 & 0\end{array}\right]^{T}$ respectively. Therefore a trajectory 


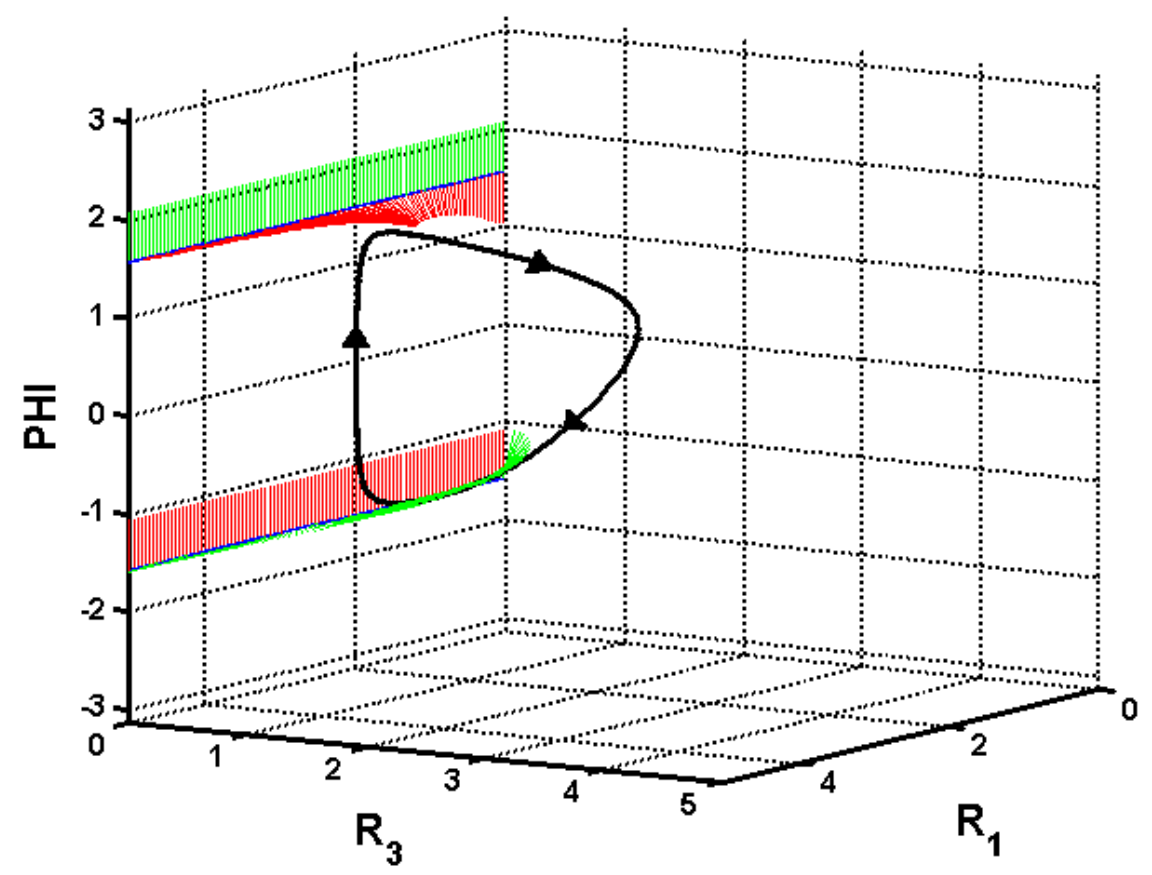

Figure 5.5: Example of stable LCW when $(\Delta, \mu)=(0.2,0.304)$

can only enter or exit the singular surfaces from the planes $\phi= \pm \frac{\pi}{2}$. Furthermore, the original in-phase slow-flow equation governing the flow in the $\phi$-direction (3.3c) becomes

$$
\frac{d \phi}{d \eta}=\frac{\mu}{2}\left(\frac{2 R_{1}}{R_{3}}-\frac{R_{3}}{R_{1}}\right) \cos (\phi)
$$

showing that the planes $\phi= \pm \frac{\pi}{2}$ are invariant, meaning that a trajectory with an initial condition on either of these planes will stay on that plane for all time. Thus the phase-space can be considered to be divided into 2 distinct regions. We leave the discussion here as the significance of these special circumstances is beyond the scope of this thesis. 


\section{CHAPTER 6 CONCLUSIONS}

In this thesis we have begun to investigate the dynamical behavior of a system of three weakly-coupled van der Pol oscillators. From our biological motivation an indirect coupling scheme was envisioned, with two identical eye oscillators coupled via a third oscillator representing the extra-ocular circadian system of the Japanese quail. Of particular interest to us were the existence of in-phase motions of the eye oscillators that are phase-locked to those of the extra-ocular oscillator.

Using singular perturbation methods (the method of two-variable expansion and the method of averaging), we reduced the four-dimensional in-phase subspace problem (2.10) to a three-dimensional slow-flow (3.3). With computational tools we successfully computed the bifurcation curves (3.11) and (3.19) for the in-phase subspace (see Figure 6.1). Furthermore, the qualitative behavior of each distinct region in the parameter space was determined and three main types of dynamical behavior were found - phase-locked motion, weakly phase-locked motion, and phase-drift. 


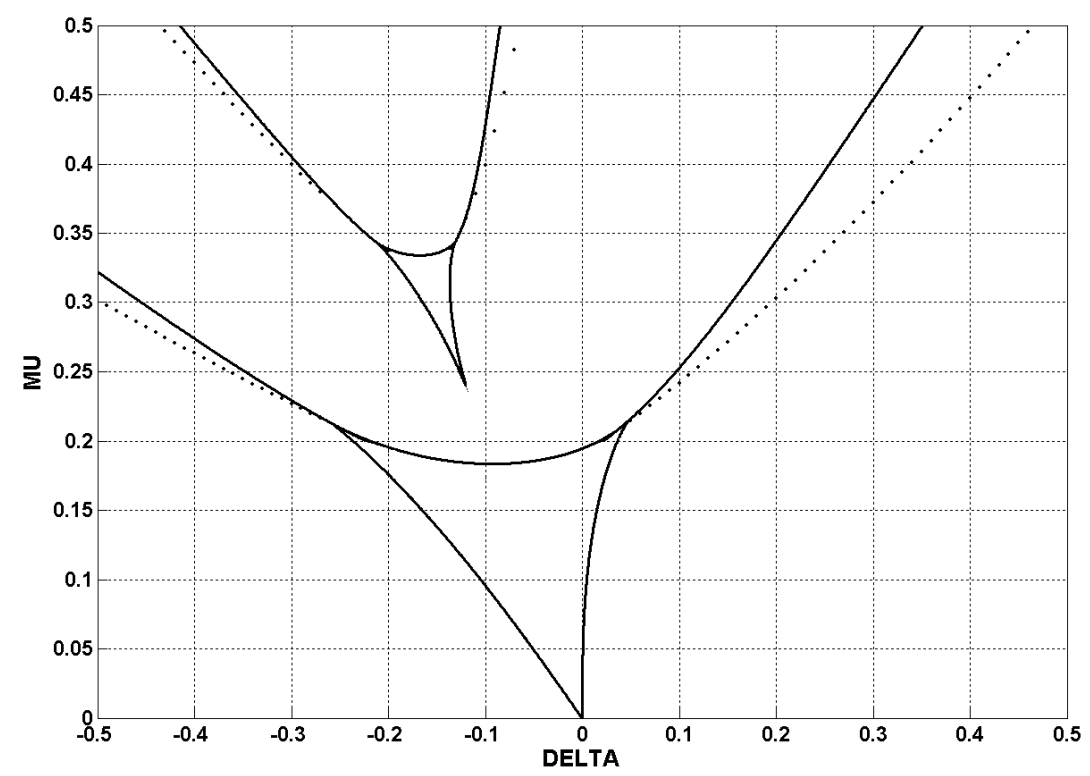

Figure 6.1: Bifurcation curves in parameter space.

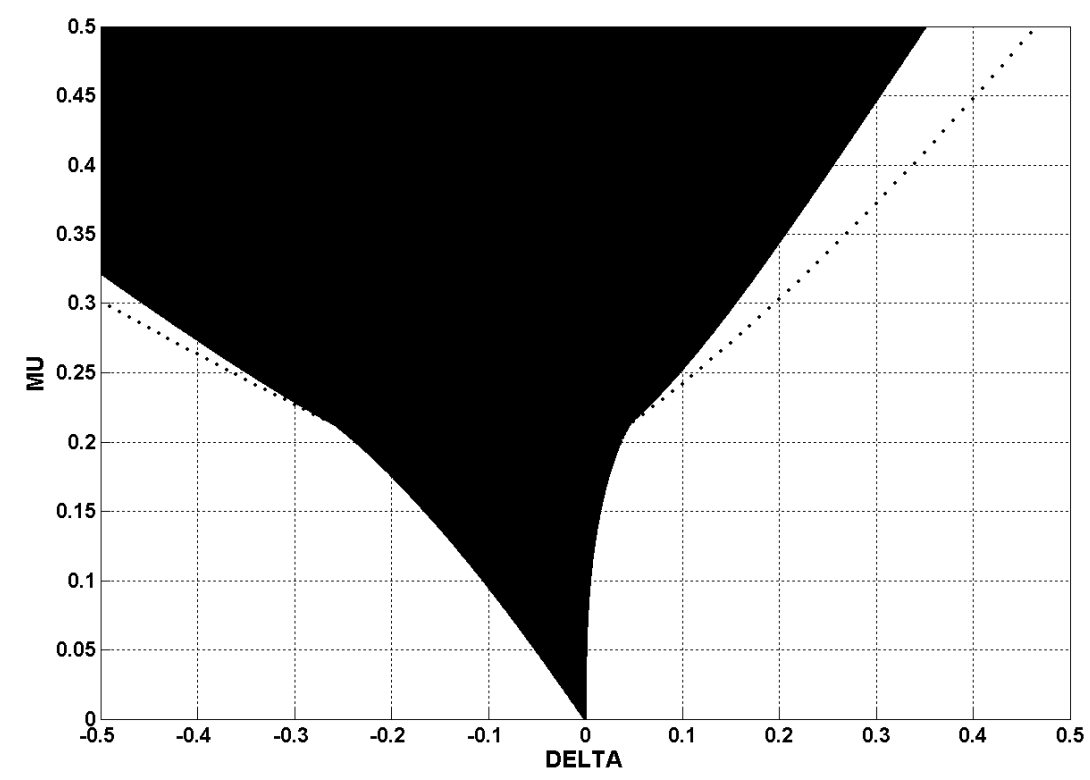

Figure 6.2: Regions of parameter space where stable phase-locked motion is possible. 
Of biological importance is the existence of stable equilibria in the slow-flow (found in Regions III, IV, V, VI, B, and C as shown in Figure 6.2). These equilibrium points correspond to stable phase-locked motions of the original four-dimensional in-phase problem (3.1). The use of linear coupling as well as the restriction of $\mu>0$ (excitatory coupling) are in agreement with previous work [2]. It is interesting to note that the regions of bi-stable dynamical behavior generally occur for $\Delta<0$. This corresponds to the eye oscillators having a higher frequency (and thus shorter period) than the extra-ocular oscillator. Future work is necessary for a better understanding of the biological significance (if any) of this result. Furthermore, future work is needed to investigate whether the in-phase subspace of the original problem (2.1) is an attracting subspace and how the stability of the out-of-phase subspace relates to that of the in-phase subspace.

This work on the in-phase subspace dynamics is reminiscent of the problem of two weakly-coupled van der Pol oscillators [4], [5]. In those works the same three types of dynamical behavior were present and the mechanism of bifurcation between phase-drift (LCD) and weakly phase-locked motion (LCW) was first elucidated. The resulting bifurcation diagram for two weakly-coupled van der Pol oscillators is shown in Figure 6.3. We see that the addition of a third van der Pol oscillator (with indirect coupling) has destroyed the symmetry of the bifurcation diagram and created a second, smaller triangular region for $\Delta<0$.

Due to symmetry in the problem of two weakly-coupled van der Pol oscillators, everywhere in Figure 6.3 where there is one stable equilibrium point, $\left(R_{1}, R_{2}, \phi\right)=$ $\left(\bar{R}_{1}, \bar{R}_{2}, \bar{\phi}\right)$, there is a second one at $\left(R_{1}, R_{2}, \phi\right)=\left(\bar{R}_{2}, \bar{R}_{1}, \bar{\phi}+\pi\right)$. Therefore all 
regions of the parameter space where there exist phase-locked motions are regions of bi-stability (Figure 6.4). However, from the description of the in-phase dynamics given in Chapter 4 we see that the regions of bi-stability have decreased and been shifted upwards (Figure 6.5).

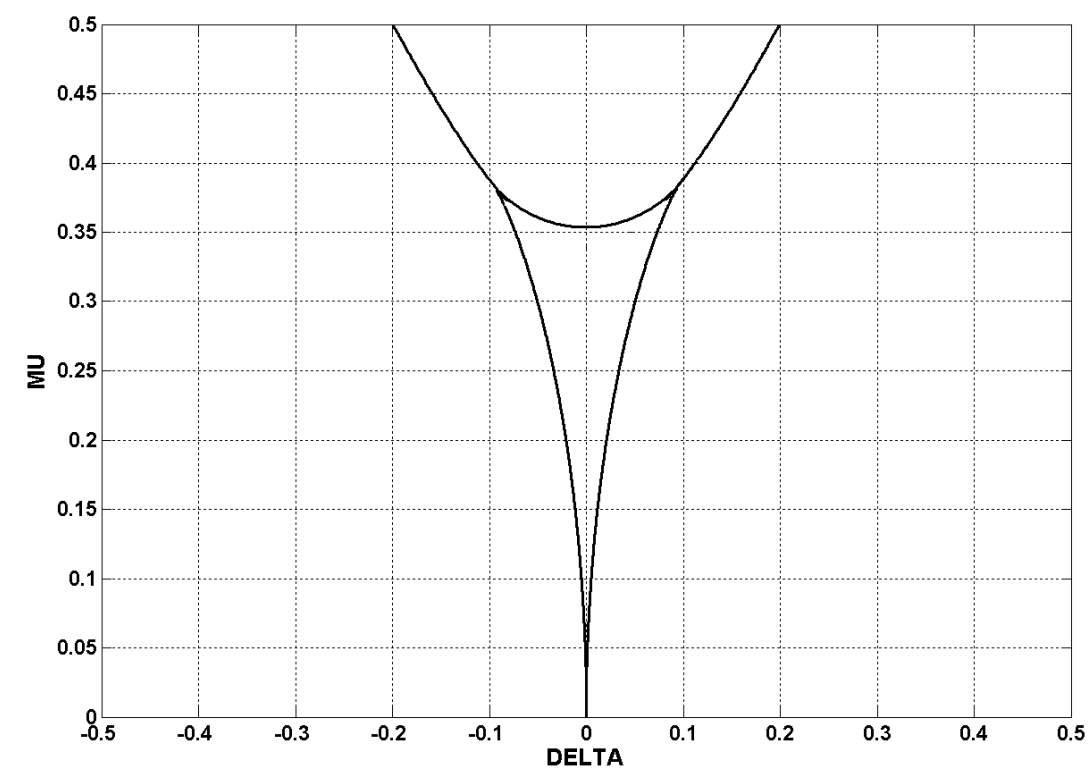

Figure 6.3: Bifurcation curves for two weakly-coupled van der Pol oscillators (modified from [4], [5])

This raises the important questions of where the new triangular region originated from and how the problems of two weakly-coupled van der Pol oscillators and three indirectly weakly-coupled oscillators are mathematically connected. While a theoretical investigation of these questions is beyond the scope of this thesis, some simple numerical investigations provide some insight. 


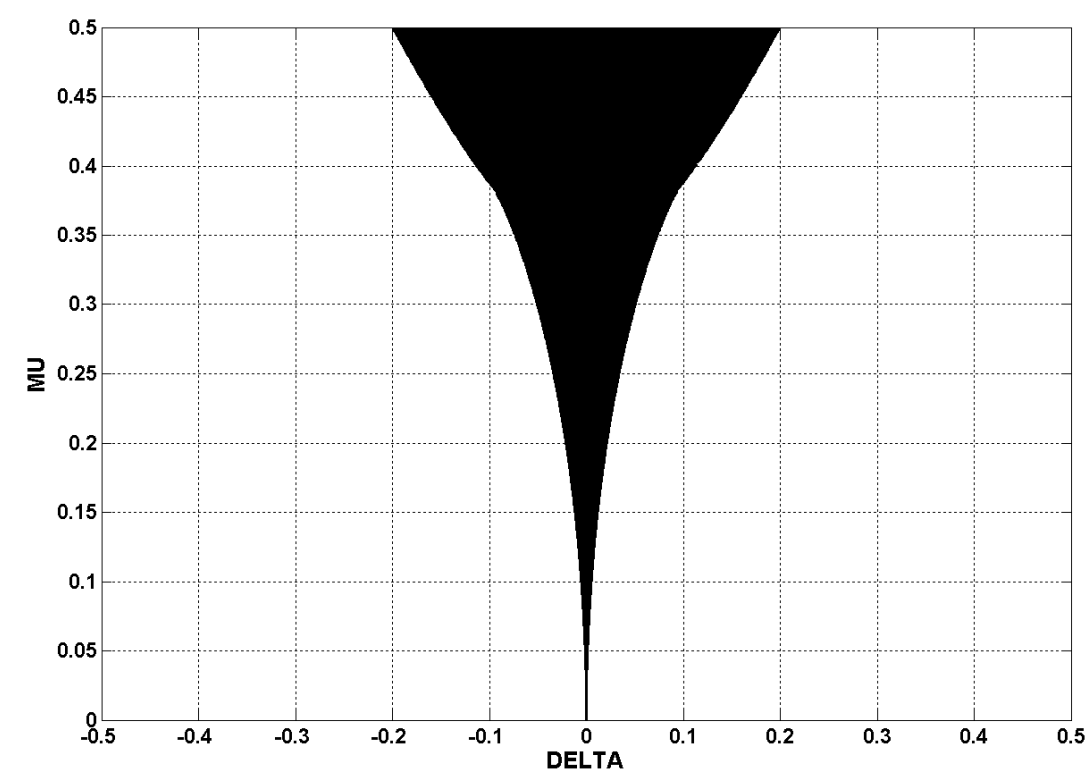

Figure 6.4: Regions of bi-stability (shown in black) for two weakly-coupled van der Pol oscillators

The equations of motion for two weakly-coupled van der Pol oscillators are

$$
\begin{gathered}
\ddot{x}-\epsilon\left(1-x^{2}\right) \dot{x}+x=\epsilon \mu(w-x) \\
\ddot{w}-\epsilon\left(1-w^{2}\right) \dot{w}+p^{2} w=\epsilon \mu(x-w)
\end{gathered}
$$

We see that equations (6.1) are similar to (3.1), the equations of motion governing the in-phase mode of three indirectly-coupled van der Pol oscillators, except for a missing factor of 2 in the $w$-equation. Thus we now generalize (3.1), writing the governing equations for the in-phase mode of $N$ van der Pol oscillators indirectly coupled via a central oscillator as

$$
\begin{gathered}
\ddot{x}-\epsilon\left(1-x^{2}\right) \dot{x}+x=\epsilon \mu(w-x) \\
\ddot{w}-\epsilon\left(1-w^{2}\right) \dot{w}+p^{2} w=\epsilon N \mu(x-w)
\end{gathered}
$$




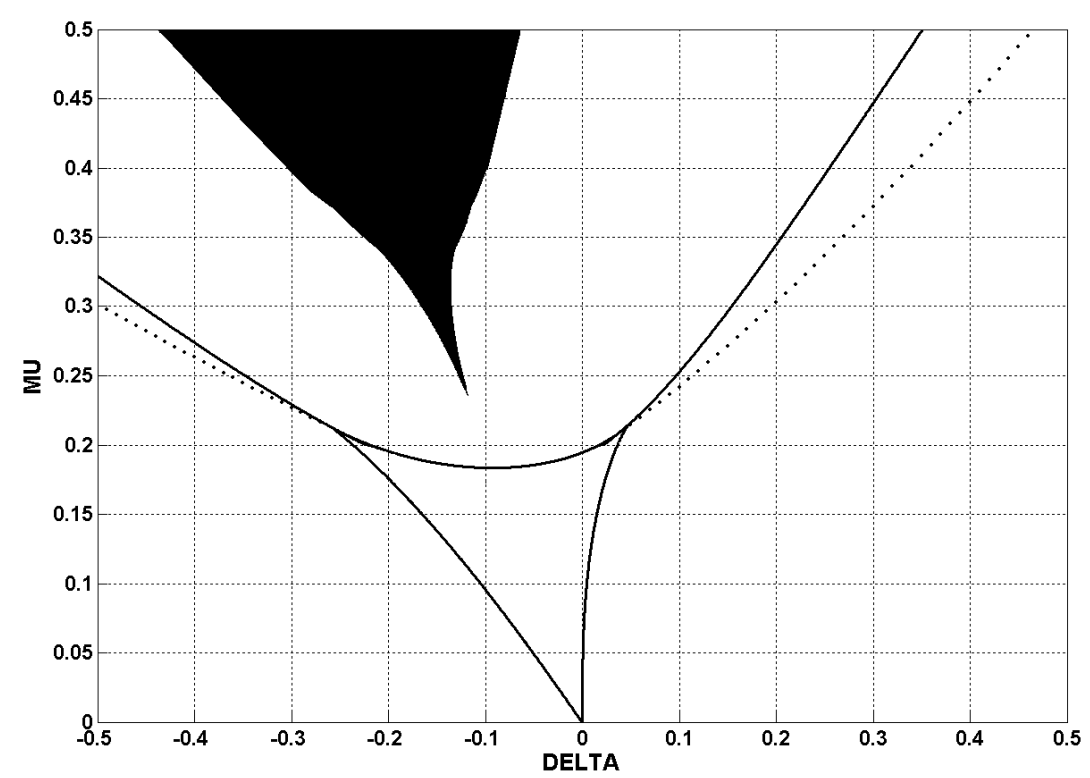

Figure 6.5: Regions of bi-stability (shown in black) for the slow-flow (3.3).

We note that setting $N=1$ in (6.2) gives the equations (6.1) for two weaklycoupled van der Pol oscillators and setting $N=2$ gives the equations for the inphase mode of three indirectly coupled van der Pol oscillators (3.1). We can now investigate, for example, how the saddle-node bifurcation curves (3.11) change as $N$ is continuously changed from 2 to 1 . Note, however, that allowing $N$ to take on non-integer values turns the problem into a purely mathematical one, as it makes no sense to consider fractions of oscillators.

Figure 6.6 shows the saddle-node bifurcation curves for different values of $N$. We see that as $N$ decreases from 2, the upper triangular region increases in size and moves towards the origin. At the same time, the lower triangular region straightens out towards the $\mu$-axis. When $N=1$ the regions coincide symmetrically about the $\mu$-axis. We note that this is not a conclusive result, but indicates that further 
theoretical investigations of equations (6.2) are warranted.
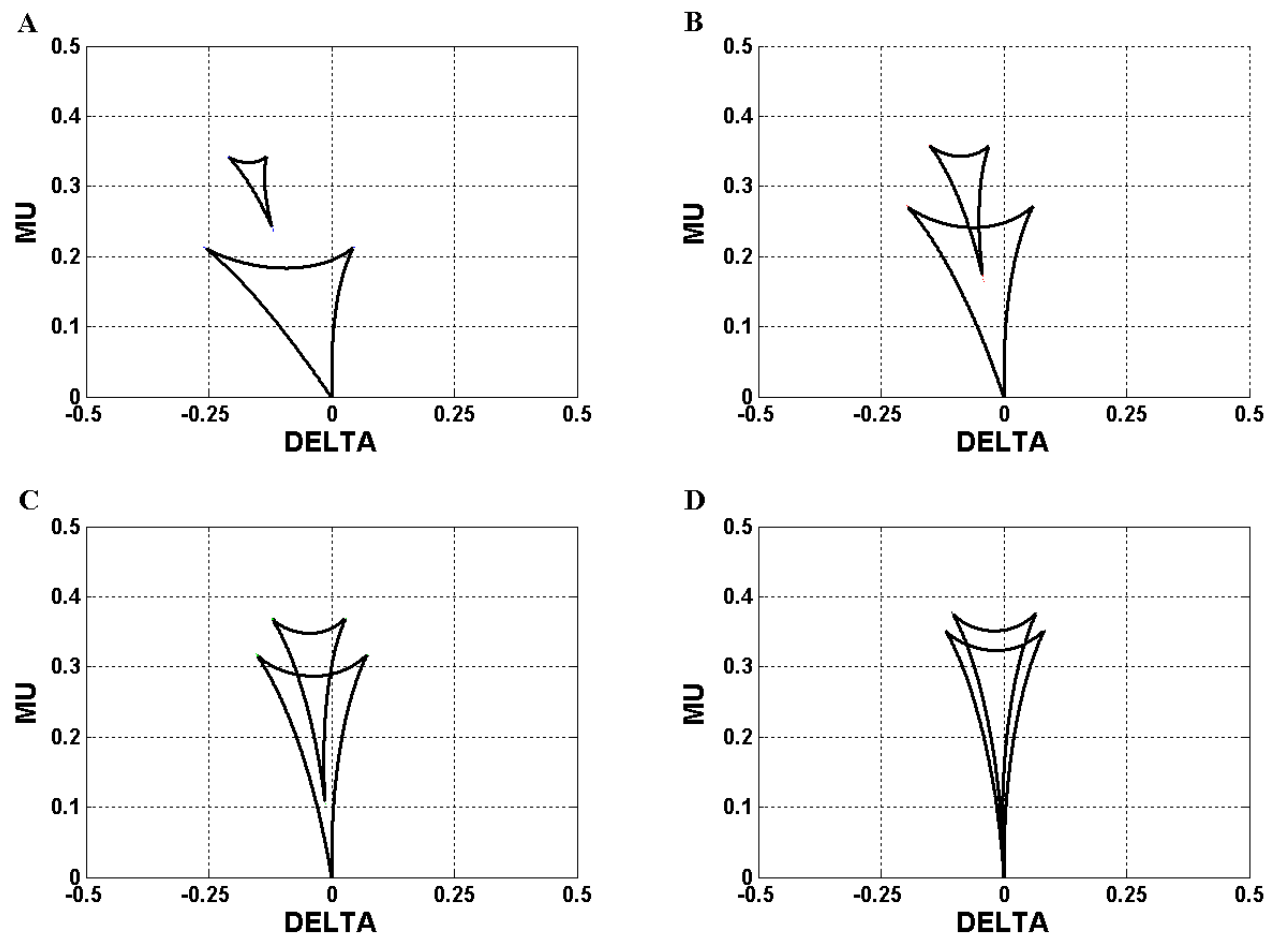

Figure 6.6: Saddle-node bifurcation curves in parameter space associated with equation (6.2) for different values of $N$ : (A) $N=2$, (B) $N=1.5$, (C) $N=1.25,(\mathrm{D}) N=1.1$. 


\section{BIBLIOGRAPHY}

[1] Camacho, E.T., "Mathematical Models of Retinal Dynamics", Doctoral thesis, Cornell University, 2003.

[2] Camacho, E.T., Rand, R.H., Howland, H., "Dynamics of two van der Pol oscillators coupled via a bath". Int. J. Solids Struct., vol. 41, pg. 2133-2143, 2004.

[3] Cassone, V., Menaker, M., "Is the Avian Circadian System a Neuroendocrine Loop?". J. Exp. Zoo., vol. 232, pg. 539-549, 1984.

[4] Chakraborty, T., "Bifurcation analysis of two weakly coupled Van der Pol oscillators". Doctoral thesis, Cornell University, 1986.

[5] Chakraborty, T. and Rand, R.H., "The transition from phase locking to drift in a system of two weakly coupled van der Pol oscillators". Int. J. Nonlinear Mech., vol. 23, pg. 369-276, 1988.

[6] Gwinner, E., "Melatonin in the Circadian System of Birds: Model of Internal Resonance". In: Hiroshige, T., Honma, K. editors. Circadian clocks and ecology. Saporo, Japan: Hokkaido Univ. Press, pg. 127-145, 1989.

[7] Janik, D., Dittami, J., Gwinner, E., "The Effect of Pinealectomy on Circadian Plasma Melatonin Levels in House Sparrows and European Starlings". J. Biol. Rhythms, vol. 7, pg. 277-286, 1992.

[8] Rand, R.H., Topics in Nonlinear Dynamics with Computer Algebra. Gordon and Breach Science Publishers, 1994.

[9] Rand, R.H., Lecture notes in nonlinear vibrations (version 45). Ithaca, NY: The Internet-First University Press; 2004. Available from: http://dspace.library.cornell.edu/handle/1813/79.

[10] Steele, C.T., Zivkovic, B.D., Sipoes, T., and Underwood, H., "Ocular clocks are tightly coupled and act as pacemakers in the circadian system of Japanese quail". Am. J. Physiol. Regul. Integrat. Comp. Physiol., vol. 284, pg. R208R218, 2003.

[11] Strogatz, S.H., Nonlinear Dynamics and Chaos: With Applications to Physics, Biology, Chemistry, and Engineering. Perseus Books, Cambridge, Massachusetts, 1994.

[12] Underwood, H., Edmonks, K., "The circadian rhythm of thermoregulation in Japanese quail. III. Effects of melatonin administration. J. Bio. Rhy., vol. 10, pg. 284-298, 1995. 
[13] Underwood, H., Steele, C.T., and Zivkovic, B., "Circadian Organization and the Role of the Pineal in Birds". Microscopy Research and Technique, vol. 53, pg. 48-62, 2001. 